\title{
SOD1 activity thresholds and TOR signalling modulate VAP(P58S) aggregation via ROS-induced proteasomal degradation in a Drosophila model of Amyotrophic Lateral Sclerosis
}

\author{
Kriti Chaplot $^{1}$, Lokesh Pimpale ${ }^{1, a}$, Balaji Ramalingam², \\ Senthilkumar Deivasigamani ${ }^{1, \mathrm{~b}}$ Siddhesh S. Kamat ${ }^{1}$, Girish S. Ratnaparkhi ${ }^{1, *}$ \\ ${ }^{1}$ Department of Biology, Indian Institute of Science Education \& Research, Pune \\ 411008, INDIA \\ ${ }^{2}$ Oxford Nanoimaging Ltd, Oxford, UK
}

*Author for Correspondence: girish@iiserpune.ac.in

\section{Present Address}

${ }^{a} B i o t e c h n o l o g y$ Center, Technische Universität Dresden, Tatzberg 47/49, 01307

Dresden, Germany

${ }^{b}$ Neurobiology and Epigenetics Unit, European Molecular Biology Laboratory, Adriano Buzzati-Traverso Campus, Via Ramarini 32, 00015 Monterotondo (RM), Italy.

Running Title: SOD1 and mTOR regulate VAPB aggregation via ROS 


\section{Abstract}

Familial Amyotrophic Lateral Sclerosis (F-ALS) is an incurable, late onset motor neuron disease, linked strongly to various causative genetic loci. ALS8 codes for a

4 missense mutation, P56S, in VAMP-associated Protein B (VAPB) that causes the protein

5 to misfold and form cellular aggregates. Uncovering genes and mechanisms that affect aggregation dynamics would greatly help increase our understanding of the disease and

7 lead to potential therapeutics.

Here, we develop a quantitative high-throughput, Drosophila S2R+ cell-based kinetic assay coupled with fluorescent microscopy to score for genes involved in the modulation of aggregates of fly ortholog, $\operatorname{VAP}(\mathrm{P} 58 \mathrm{~S})$, tagged with GFP. As proof of principle, we conducted a targeted RNAi screen against 900 genes, consisting of VAP genetic interactors, other ALS loci, as also genes involved in proteostasis. The screen identified 150 hits that modify aggregation, including the ALS loci SOD1, TDP43 and also genes belonging to the TOR pathway.

To validate these modifiers, we developed a system to measure the extent of VAP(P58S) aggregation in the Drosophila third instar larval brain using the UAS-GAL4 system, followed by quantitative imaging of cellular inclusions. We find that reduction of SOD1 activity or decreased TOR signalling reduces aggregation. Interestingly, we find that increase in cellular reactive oxygen species (ROS) levels, assessed by measuring oxidation of cellular lipids and proteins, in response to SOD1 knockdown or by inhibition of TOR signalling appears to be the trigger for clearing of aggregates. The mechanism of aggregate clearance is, primarily, the proteasomal machinery, and not autophagy. Increase in VAP, but not VAP(P58S) levels, appears to elevate ROS, which may in turn regulate VAP transcription in a feedback loop.

We have thus uncovered an interesting interplay between SOD1, ROS and TOR signalling that regulates the dynamics of VAP aggregation. Mechanistic processes

27 underlying such cellular regulatory networks will lead us to a better understanding of initiation and progression of ALS. 


\section{Keywords}

32 VAP, VAMP-associated Protein B; SOD1, Superoxide dismutase; mTOR or TOR, mechanistic Target of Rapamycin; ALS, Amyotrophic Lateral Sclerosis; ROS, Reactive Oxygen Species, PS, phosphatidylserine, PE, phosphatidylethanolamine; PUFA, polyunsaturated fatty acids; UPS, Ubiquitin Proteasomal System; ERAD, Endoplasmic Reticulum Associated Degradation.

\section{Introduction}

Amyotrophic lateral sclerosis (ALS) is a progressive, fatal neurodegenerative disease characterized by loss of motor neurons resulting in muscular atrophy, gradual paralysis and ultimately death of the patient within 2-5 years post diagnosis (CLEVELAND AND ROTHSTEIN 2001; TARASIUK et al. 2012). Most often, the disease occurs sporadically (S-ALS). However, in $\sim 10 \%$ of the cases, the disease occurs due to inheritance of altered gene(s) (F-ALS). ALS1/SOD1 coding for superoxide dismutase 1, was the first causative locus to be discovered (DENG et al. 1993; Rosen et al. 1993), with more than 170 SOD1 mutations attributed to the diseased state. Since then, about 50 potential genetic loci (TAYLOR et al. 2016) have been identified in ALS through Genome-wide association (GWAS), linkage and sequencing studies. Recent studies have emphasized on the oligogenic basis for ALS (VAN BLITTERSWIJK et al. 2012; DEIVASIGAMANI et al. 2014), suggesting that ALS loci may be a part of a gene regulatory network (GRN) that breaks down late in the life of a diseased individual. At the cellular level, several hallmarks of ALS include breakdown of cellular homeostasis (CLUSKEY AND RAMSDEN 2001), endoplasmic reticulum (ER) stress, unfolded protein response, aggregation, oxidative stress, mitochondrial dysfunction and autophagy. While several studies have

57 function, no clear unifying mechanism has emerged that might explain the pathogenesis

58 of the disease (ANDERSEN AND Al-ChalABI 2011; WALKER AND ATKIN 2011; MULLIGAN AND 59 ChAKRABARTTY 2013; TURNER et al. 2013; TAYLOR et al. 2016).

62 family whose members succumbed to ALS and/or Spinal muscular atrophy (SMA). The 
63 point mutation of P56S was identified in the N-terminal, Major Sperm Domain (MSP) of

64 VAPB (NISHIMURA et al. 2004). VAPB is an integral membrane protein present in the ER

65 membrane, ER-Golgi intermediate compartment, mitochondrial-associated membrane

66 and the plasma membrane, implicated in important functions in the cell such as vesicular

67 trafficking, ER structure maintenance, lipid biosynthesis, microtubule organization,

68 mitochondrial mobility and calcium homeostasis (LEV et al. 2008; MURPHY AND LEVINE

69 2016). Recent studies have highlighted its critical role in membrane contact sites (ALPY

70 et al. 2013; GomeZ-Suaga et al. 2017b; MetZ et al. 2017; YAdAV et al. 2018; ZhAO et al.

71 2018). The Drosophila ortholog of VAPB is VAP33A/CG5014 (Called VAP hereafter) and

72 has been used to develop models for ALS (CHAl et al. 2008; RATNAPARKHI et al. 2008;

73 Deivasigamani et al. 2014; Moustaqim-BarRette et al. 2014; SanhuezA et al. 2015). We

74 have previously identified a Drosophila VAP GRN comprising of 406 genes, including a

75 novel interaction with the mTOR pathway (DEIVASIGAMANI et al. 2014). The ALS8 mutation

76 can also alter VAP's physical interaction with other proteins, including FFAT motif

77 containing proteins (LOEWEN et al. 2003; MURPHY AND LEVINE 2016), impairing cellular

78 functions (DE Vos et al. 2012; MoustAqIM-BARRETTE et al. 2014; HutTLIN et al. 2015).

79 Ubiquitinated cellular aggregates (RATNAPARKHI et al. 2008; PAPIANI et al. 2012) are seen

80 on VAP mutant expression, and are capable of sequestering the wildtype VAP protein in

81 a dominant negative manner (TEULING et al. 2007; RATNAPARKHI et al. 2008). In

82 Drosophila, neuronal overexpression of VAP(P58S), and subsequent formation of

83 aggregates, in the background of endogenous VAP appear to lead to only mild

84 neurodegenerative phenotypes, such as flight defects, as compared to the more severe

85 phenotypes associated with wild type VAP neuronal overexpression (RATNAPARKHI et al.

86 2008; TSUDA et al. 2008). Previously, we have used the UAS-GAL4 system to study the

87 interaction between VAP and mTOR signalling using the NMJ phenotype associated with

88 neuronally overexpressed VAP(P58S)(DEIVASIGAMANI et al. 2014). The functional

89 consequence of neuronal VAP(P58S) aggregation in this system is not fully understood

90 and its contribution to disease remains elusive.

91 In this study, we identify 150 genetic modifiers of VAP(P58S) aggregation by

92 conducting a directed S2R+ cell based RNAi screen, targeting 900 unique genes

93 belonging to different categories that are associated either with ALS or VAP function or 
94 proteostasis. We used the previously described (C155-Gal4;UAS-VAP(P58S)) system 95 (RATNAPARKHI et al. 2008; DeIVASIGAMANI et al. 2014) to validate one such modifier, SOD1,

96 in vivo, in the third instar larval brain of Drosophila by measuring changes in aggregation 97 of $\operatorname{VAP}(\mathrm{P} 58 \mathrm{~S})$ in response to modulation of SOD1 levels. Our data indicates that 98 clearance of $\operatorname{VAP}(\mathrm{P} 58 \mathrm{~S})$ aggregates via the proteasomal machinery is enhanced by 99 inducing reactive oxygen species (ROS) due to loss of SOD1 function. We also find a 100 similar clearance of aggregation, attributed to proteasomal degradation, with mTOR 101 downregulation accompanied by elevated ROS. We find that wild type VAP, but not 102 mutant VAP, elevates ROS. Accumulated ROS results in inhibition of endogenous VAP 103 transcription, a phenomenon that may directly affect both familial as well as sporadic ALS 104 pathogenesis. 


\section{Results}

A Drosophila S2R+ cell culture model to study VAP(P58S) aggregation

C-terminal and N-terminal fusions of VAP and VAP(P58S) with GFP were used to transfect cells and generate stable S2R+ lines, as described in Materials \& Methods (Fig. 1A, Suppl. Fig. 1A). VAP:GFP showed a non-nuclear, reticular localization in the cell with $<10 \%$ of the transfected (GFP-positive) cells showing high intensity puncta (Fig. 1B, Suppl. Fig. 1A). In contrast, $>80 \%$ of the GFP-positive VAP(P58S):GFP, cells showed distinct high intensity puncta with little or no background staining within the cell (Fig. 1C, Suppl. Fig. 1A). Super resolution imaging confirmed that VAP appeared to be reticular, while VAP(P58S) was found in inclusion bodies (Fig. 1D). In contrast, GFP, when expressed showed a uniform cytoplasmic signal (Suppl. Fig. 1B). Both N-terminal GFP fusions, GFP:VAP and GFP:VAP(P58S) showed puncta formation at levels comparable to VAP(P58S):GFP, and hence were not used further in the study (Suppl. Fig. 1A). All further experiments (see next section) were carried out with stable lines expressing VAP:GFP or VAP(P58S):GFP, which showed expected/relevant localization and levels of aggregation.

An S2R+ cell based reverse genetics screen is developed to identify modifiers of VAP(P58S) aggregation

In an attempt to identify genetic modifiers of VAP(P58S) aggregation kinetics, we conducted a focused S2R+ cell based RNAi screen, targeting 900 unique genes belonging to nine different categories or families associated with ALS or VAP function. We generated stable S2R+ cell lines expressing VAP(P58S):GFP under a $\mathrm{Cu}^{2+}$ induced promoter. The inducible cell culture system allowed us to increase the VAP(P58S):GFP protein levels in the cell with increasing copper sulphate $\left(\mathrm{CuSO}_{4}\right)$ concentrations $(250 \mu \mathrm{M}$, $500 \mu \mathrm{M}, 750 \mu \mathrm{M}$ and $1000 \mu \mathrm{M})$ at 24 hours post induction (Fig. 1E). Using fluorescence microscopy, we found a linear relationship between the copper sulphate $\left(\mathrm{CuSO}_{4}\right)$ concentrations and also the fraction of cells showing VAP(P58S):GFP aggregates that also increased with time (24 and 36 hours) post induction (Fig. 1F). The concentration dependent increase in relative levels of VAP(P58S):GFP correlated with an increase in fraction of cells showing aggregates (Fig. 1G), indicating the propensity of the mutant 
138 protein to aggregate. Early time points (12-16 hours) gave very few cells with aggregates;

139 while non-linearity, high confluency, and cell death became a concern at time points 140 beyond 48 hours and concentrations greater than $750 \mu \mathrm{M}$. The aggregation kinetics 141 curve was used to define the extent of aggregation in the cell culture system and select 142 optimum parameters to conduct the RNAi screen. Keeping a modest confluency and well143 separated cells for ease of imaging, the screen was performed at a fixed concentration 144 of $500 \mathrm{\mu M} \mathrm{CuSO}_{4}$ at 24 and 36 hours post induction.

145 We chose 900 genes (Suppl. Table 1A), based on their availability in the Open Biosystems Library (See Materials \& Methods) to screen for modifiers that could change 147 aggregation levels of VAP(P58S):GFP. A Gene Ontology (GO) chart (Fig. 2A) represents 148 the biological process associated with these 900 genes, as defined by FlyBase. The 149 genes were selected and categorized (Suppl. Table 1B) on the following basis. First, known Drosophila Orthologs of ALS loci (20 genes) and ALS related genes (36 genes) as tabulated in the online ALS database (ALSOD) were chosen. The next category included 273 genes from a VAP Drosophila GRN comprising of 406 genes (DEIVASIGAMANI et al. 2014). As mTOR was identified as a major interactor of VAP in our previous study 154 (DeIVAsigamani et al. 2014), we chose 22 genes of the extended mTOR pathway. To 155 explore the functional aspects of VAP(P58S), we also screened genes involved in lipid biosynthesis (92 genes) and FFAT motif interactors of VAP (34 genes). In order to identify 157 a role of proteostasis in aggregation, we screened genes involved in unfolded protein 158 response (123 genes), ubiquitin-proteasomal pathway (212 genes), and autophagy (88 genes)

The images collected at the end of the screen (detailed in Materials and Methods) 162 Based on average cell intensity, 150 targets (Suppl. Table 1C), and based on total cell 163 intensity, 85 targets (Suppl. Table 1D) that modulated VAP(P58S):GFP aggregation 164 kinetics were identified; 55 genes were found to be targets as per both parameters. 165 Enrichment profile of target genes are plotted in Fig 2C and Suppl. Fig. 1C. ALS loci, 166 notably SOD1 and TBPH, were found as interesting modulators perturbing 167 VAP(P58S):GFP aggregation. Targets belonging to the VAP genetic network, as defined 168 by (DEIVASIgAmANI et al. 2014), were also enriched. As identified earlier (DEIVASIGAMANI et 
al. 2014), components of the mTOR pathway also appeared to be key regulators of VAP(P58S):GFP aggregation. However, less than $10 \%$ of genes screened belonging to families associated with lipid biosynthesis and motif interactors, were identified as targets,

172 suggesting lower functional relevance for VAP(P58S):GFP. Interestingly, genes related

173 to ubiquitin proteasomal system such as ubiquitin ligases and proteasome components 174 were enriched, as were the autophagy related genes such as ATG7 and ATG3. From the 175 unfolded protein response category, along with chaperones such as heat shock proteins, 176 we also identified peptidyl prolyl isomerases as targets. Overall, in our primary targeted 177 screen, we found various genetic interactors of wildtype VAP as modulators of 178 VAP(P58S) aggregation as well; importantly, the uncovering of two ALS loci, SOD1 and 179 TDP-43, mTOR pathway genes such as Rheb and S6K, and genes enriched in ubiquitin proteasomal system as modulators of VAP(P58S) aggregation dynamics, lead us to develop an in vivo model to validate these genes and to understand mechanisms underlying these interactions in the animal.

A model system for measuring VAP(P58S) aggregation in the Drosophila larval brain.

In order to validate targets from the screen in vivo, we used the UAS-GAL4 system to specifically overexpress wild-type $V A P$ or $V A P(P 58 S)$ in the brain using a pan-neuronal driver, C155 (elav) (RATNAPARKHI et al. 2008; DEIVASIGAMANI et al. 2014). Based on antiVAP immunostaining, unlike wild-type VAP (Suppl. Fig. 2A), mutant VAP(P58S) formed distinct cellular puncta and could be used as a model to study aggregation in the animal (Suppl. Fig. 2B-D). These aggregates have been shown to be ubiquitinated and dominant-negative when expressed in muscle (Ratnaparkhi et al, 2008). To develop a 192 methodology for quantitation of aggregates in the brain (described in Materials \& 193 Methods), we used temperature as a means to increase GAL4 activity, which would 194 increase VAP(P58S) dosage and possibly, aggregation. An increase in mean VAP(P58S) 195 aggregation density was observed from $18^{\circ} \mathrm{C}$ to $25^{\circ} \mathrm{C}$, but not significantly between 25 $196{ }^{\circ} \mathrm{C}$ and $28^{\circ} \mathrm{C}$ (Suppl. Fig. 2H). Neuronal knockdown of VAP, using RNAi, in CI55-GAL4/+; 197 UAS-VAP(P58S)/+ flies, at each temperature (Suppl. Fig. 2E-G), led to a significant 198 decrease in corresponding aggregation density of the ventral nerve cord (Suppl. Fig. 2H). 199 The above experiments suggest that at $25^{\circ} \mathrm{C}$, we could quantify changes in VAP(P58S) 
200 aggregation density in the brain of the larvae, and here onwards, we use this system to

201 further validate modifiers of aggregation identified from the cell-based screen.

202

Drosophila SOD1 is a modifier of VAP(P58S) aggregation

SOD1, first known ALS locus (RosEN et al. 1993), has been implicated in both sporadic as well as familial cases and was our first choice for validation of the S2R+ based screen, in the animal. We previously identified SOD1 as a genetic interactor of VAP in a fly-based reverse genetics screen (DEIVASIGAMANI et al. 2014). Here, we individually knocked down SOD1 using three independent RNAi lines in the CI55-GAL4/+; UAS-VAP(P58S)/+ background and observed a significant decrease in aggregation density in the ventral nerve cord (Fig. 3A, 3B, Suppl. Fig. 3A, 3C, 3D). This three-fold decrease in VAP aggregates was comparable to the reduction seen with VAP RNAi. Likewise, we overexpressed SOD1 in the CI55-GAL4/+; UAS-VAP(P58S)/+ background. Here, however, we did not find a significant change in aggregation density (Fig. 3C, 3D

214 Suppl. Fig. 3B, 3C, 3E). Taken together, these results suggest a need for a threshold 215 level of SOD1 to maintain VAP(P58S) inclusions.

Oxidative stress reduces VAP(P58S) aggregation

Enzymatically, SOD1 metabolizes superoxide species to hydrogen peroxide, thereby preventing oxidative stress. A loss of function of SOD1 would, in principle, increase ROS. We tested whether a chemical mimic, paraquat, which increases cellular ROS (CAstello et al. 2007; DReChSEl ANd PATEl 2008; Cocheme et al. 2011), could phenocopy the effect of SOD1 knockdown. We treated the VAP(P58S):GFP stable line with non-lethal concentrations of $10 \mathrm{mM}$ and $20 \mathrm{mM}$ paraquat for 4 hours prior to $\mathrm{CuSO}_{4}$ induction and found that paraquat could significantly reduce the fraction of cells showing 225 GFP positive aggregates (Fig. 4A, Suppl. Fig. 4A) in a dose-dependent manner. Similarly, 226 larvae with the genotype CI55-GAL4/+; UAS-VAP(P58S)/+ hatched, fed and grown on 227 non-lethal concentration of $5 \mathrm{mM}$ paraquat at $25^{\circ} \mathrm{C}$, showed a decrease in aggregation 228 density in the third instar larval brain, reminiscent of the SOD1 knockdown phenotype 229 (Fig. 4B, Suppl. Fig. 4B). We also checked the effect of other ROS scavenging genes such as SOD2 and catalase on VAP(P58S) aggregation. Knockdown of both these genes 
231 resulted in a drastic reduction in aggregation density in the ventral nerve cord of C/55-

232 GAL4/+; UAS-VAP(P58S)/+ larval brains. As seen with SOD1, overexpression of SOD2

233 did not change aggregation density; however, catalase overexpression resulted in a

234 fractional increase in aggregation density (Suppl. Fig 3F). These results strongly suggest

235 a ROS dependent maintenance and/or stability of VAP(P58S) aggregates.

To confirm whether feeding of paraquat and loss of SOD1 function led to an increase in ROS levels in the larval brain, we measured the levels of oxidized proteins and lipids, using the oxyblot kit and quantitative mass spectrometry based lipidomics, respectively. Using the oxyblot assay, we found that feeding C155-GAL4/+ larvae with increasing concentrations of paraquat $(0 \mathrm{mM}, 0.05 \mathrm{mM}, 0.5 \mathrm{mM}, 5 \mathrm{mM})$ was sufficient to increase ROS in the brain, observed as an increase in intensity of oxidized proteins as compared to unfed larvae (Suppl. Fig. 4C). As expected, neuronal knockdown of SOD1 in presence of VAP(P58S) aggregates, led to a corresponding increase in intensity of oxidized proteins, demonstrating oxidative stress (Fig. 4C). We found that VAP(P58S) aggregation alone did not significantly change oxidized protein levels as compared to the C155-GAL4/+ control (Fig. 4C). Unexpectedly, we found that overexpression of VAP in neurons led to a distinct increase in oxidation of proteins (Fig. 4C).

To further bolster our findings, we measured levels of oxidized phospholipids in larval brains (TYURINA et al. 2000; KAMAT et al. 2015; KORY et al. 2017). On feeding C155-

250 GAL4/+ larvae with $5 \mathrm{mM}$ paraquat, we enriched and detected 9 oxidized 251 polyunsaturated fatty acids (PUFAs), belonging to phosphatidylserine (PS) and 252 phosphatidylethanolamine (PE) (Fig. 4D, Suppl. Table 2) families of phospholipids, which 253 were significantly elevated in larval brains, compared to the unfed control. PUFA 254 containing oxidatively damaged phospholipids showed a mass addition of +16 (denoted as ox-) or +18 (denoted as hy-) to the parent phospholipid, as a consequence of addition

256 of different ROS. Of note, the parent or precursor phospholipids did not change in 257 concentration, and the concentrations of the oxidized phospholipids were less than 1\% 258 of the parent or precursor phospholipids. We found a similar elevation in concentrations 259 of oxidized phospholipids in C155-GAL4/+; UAS-VAP(P58S)/+; UAS-SOD1_i/+, but not 260 in CI55-GAL4/+; UAS-VAP(P58S)/+ which was equivalent to C155-GAL4/+ control (Fig. 261 4D, Suppl. Table 2). This elevation in oxidized phospholipids was found to be inversely 
262 correlated with corresponding fold change in aggregation density (Suppl. Fig. 4D).

263 Interestingly, we found, as suggested by the oxyblot data, overexpression of VAP had a 264 curious effect of increasing oxidation of lipids, indicating that wild type VAP has a cryptic 265 yet important role in regulating ROS levels. Taken together, these results indicate that 266 ROS initiates processes that aid clearance VAP(P58S) aggregates, and is in turn 267 regulated by VAP wildtype levels in the cell.

ROS activates proteasomal machinery

We further investigated protein degradation mechanisms that may be activated in 271 response to ROS leading to the clearance of VAP(P58S) aggregates. In order to test 272 whether the proteasomal machinery was responsible for reduction in aggregation, we 273 hatched, fed, and grew larvae with proteasomal inhibitor 5 MM MG132, and dissected the 274 brains at the wandering third instar stage and analysed the aggregation density. Unfed 275 C155-GAL4/+; UAS-VAP(P58S)/+; UAS-SOD1_i/+, as expected, showed reduced 276 aggregation density (Fig. 5C), as compared to unfed control (Fig. 5A, 5E). Upon MG132 277 feeding, C155-GAL4/+; UAS-VAP(P58S)/+; UAS-SOD1_i/+, showed a complete rescue 278 of VAP(P58S) aggregation (Fig. 5D, 5E). Fed C155-GAL4/+; UAS-VAP(P58S)/+; UAS279 SOD1_i/+ also showed an enhanced aggregation density as compared to fed C/55280 GAL4/+; UAS-VAP(P58S)/+ (Fig. 5B, 5E). Aggregates in presence of ROS (with SOD1 281 knockdown) and proteasomal inhibition (with MG132) appeared to be predominantly 282 smaller, scattered and mislocalized around the nuclear membrane/ER as compared to 283 the respective controls (Fig. 5D'). The localization of the aggregates suggest that may be 284 residing in the Juxta Nuclear Quality Control compartment (JUNQ)-like compartment 285 (OGRODNIK et al. 2014). These results indicate that the proteasomal machinery is 286 facilitated in presence of ROS for active degradation of VAP(P58S) aggregates (Fig 5F). 287 However, fed CI55-GAL4/+; UAS-VAP(P58S)/+ larvae (Fig. 5A) did not show 288 accumulation of aggregation as compared to unfed control (Fig. 5B, 5E), indicating other mechanisms may be at play to maintain the aggregation density. 
mTOR inhibition lowers VAP(P58S) aggregation but not via autophagy

We examined whether aggregates could be cleared via autophagy in the third instar larval brain. We inhibited the mTOR pathway by feeding CI55-GAL4/+; UASVAP(P58S)/+ larvae with 200nM rapamycin (HEITMAN et al. 1991) as described (DEIVASIGAMANI et al. 2014), thereby activating autophagy (NODA AND OHSUMI 1998), and observed a drastic clearance of aggregation in the ventral nerve cords as compared to unfed controls (Fig. 6A, 6B, 6C). When Tor transcripts were reduced using RNAi in Cl55GAL4/+; UAS-VAP(P58S)/+, a similar decrease in aggregation density was found (Fig. 6D, 6E, 6F). However, when autophagy was induced directly via overexpression of Atg1 in CI55-GAL4/+; UAS-VAP(P58S)/+, we did not observe clearance of aggregation (Fig. $6 \mathrm{G}, 6 \mathrm{H}, 6 \mathrm{I})$. This suggests that mTOR signalling may perturb downstream effectors other than Atg1 which may affect VAP(P58S) aggregation dynamics (Fig 6J).

mTOR inhibition promotes proteasomal clearance of VAP(P58S) aggregation via ROS

We first decided to check whether clearance of aggregates with mTOR inhibition correlated with increase in ROS, as in the case of SOD1 knockdown. We found that levels of several species of oxidized phospholipids were indeed higher with Tor knockdown with or without neuronal overexpression of VAP(P58S) in third instar larval brains to levels similar to SOD1 knockdown (Fig. 7A). mTOR pathway downregulation has recently been shown to activate not only autophagy but also ubiquitin proteasomal machinery (ZHAO et al. 2015) via Mpk1/ERK5 pathway in yeast and humans (RoussEAU AND BERTOLOTTI 2016). We tested whether ROS upregulation with Tor knockdown could be inducing proteasomal clearance of VAP(P58S) aggregation by feeding CI55-GAL4/+; UAS-

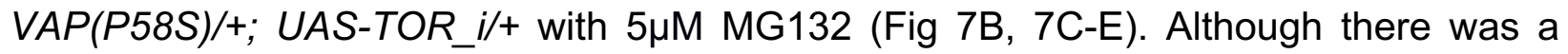
significant decrease in aggregation density with Tor knockdown (Fig. 7D), we found only a slight recovery of aggregation in MG132-fed animals (Fig. 7E) as compared to unfed CI55-GAL4/+; UAS-VAP(P58S)/+ control flies (Fig. 7C). This recovery appeared to be far less dramatic than that seen in the case of SOD1 knockdown. Taken together, these results indicate that in context of ROS, proteasomal degradation could be the major 322 pathway responsible for clearance of VAP(P58S) aggregation (Fig. 7F), although other 
323 downstream effectors of mTOR signalling, including autophagy, cannot be conclusively 324 ruled out as additional mechanisms.

325 We also explored the possible relationship between VAP and ROS at a 326 transcriptional level. Larvae of the control, CI55-GAL4/+ genotype were hatched and fed 327 on $5 \mathrm{mM}$ paraquat, and the brains were dissected at the wandering third instar larval stage.

328 The levels of endogenous VAP and SOD1 mRNA, in response to ROS, were measured 329 using qPCR in control larval brains. We found that endogenous VAP mRNA levels were 330 lower in the presence of high levels of ROS (Suppl. Fig. 4E), while SOD1 mRNA levels 331 remained unchanged (Suppl. Fig. 4F). This result may indicate the presence of a negative 332 feedback loop wherein VAP overexpression leads to accumulation of ROS (Fig. 4C-D), 333 which in turn downregulates endogenous VAP transcription.

A targeted RNAi screen uncovers SOD1, TDP43 and TOR signalling elements as targets to understand dynamics of $\operatorname{VAP}(P 58 S)$ aggregation

Drosophila S2R+ cell based whole genome RNAi screens serve as powerful tools due of the relative ease with which transcript knockdown can be achieved (ECHEVERRI AND PERRIMON 2006). Similar systems have been used for identifying modifiers of aggregation of Huntingtin protein (ZHANG et al. 2010). Our screen was aimed at enriching genes that are known players in ALS, VAP interactors and proteostasis. First and foremost, we found ALS loci, SOD1 and TDP-43 as modifiers of VAP(P58S) aggregation, which we had previously identified as VAP genetic interactors (DEIVASIGAMANI et al. 2014). In this study, we have explored the interaction between SOD1 and VAP, while TDP-43 also serves as an exciting candidate for further investigation. TDP-43 has been shown to perturb membrane-associated mitochondrial (TURNER et al. 2008) sites that are maintained by VAPB-PTPIP51 interactions in mammalian cell culture (STOICA et al. 2014). Additionally, TDP-43 proteinopathy has been identified in motor neurons of mice models

352 of VAP(P58S) aggregation (TUDOR et al. 2010). TDP-43 driven neurodegeneration has 353 also been shown to be modulated by oxidative stress related MAP kinase pathways in a 354 Drosophila screen (ZHAN et al. 2015) and associated with Nrf2 dependent antioxidant 
pathway (MOUJALLED et al. 2017). In addition to SOD1, we have also identified other ROS related genes such as peroxiredoxin V, NADH dehydrogenase, cytochrome c oxidase, that localise to the mitochondria, perturbation of which will lead to oxidative stress, potentially affecting aggregation kinetics of VAP(P58S).

Secondly, we enriched a subset of targets involved in protein degradation, UPS and autophagy, an in vivo validation of which would shed light on the how these aggregates are compartmentalized and managed in the neurons. Thirdly, this screen highlighted specific chaperones that could be involved in the misfolding and formation of VAP(P58S) aggregates providing insight into the initiation of the disease condition. Most importantly, through our previous study (DEIVASIGAMANI et al. 2014), and our cell-based screen followed by subsequent experimentation, we have established mTOR signalling as a strong modulator of VAP(P58S) aggregation. mTOR signalling responds and integrates signals from nutrients, growth factors, energy, and stress, regulates cellular proteostasis, thus contributing to age-related neurodegenerative diseases (PERLUIGI et al. 2015), making it an attractive target for further investigation in ALS pathogenesis. Indeed, rapamycin, a TORC1 inhibitor, is now being used for phase-Il clinical trials for ALS (MANDRIOLI et al. 2018). Lastly, through our screen, targeting processes involved in neurodegeneration, we have identified interactions that point towards a role for VAP as a contributor to a common gene regulatory network (GRN), in agreement with several examples in literature (TUDOR et al. 2010; VAN BLITTERSWIJK et al. 2012; PRAUSE et al. 2013; Deivasigamani et al. 2014; Stoica et al. 2014; StoicA et al. 2016; PAilLusson et al. 2017). When we compared our list of targets with the results from another fly-based screen for VAP(P58S)-induced eye degeneration (SANHUEZA et al. 2015), we found no overlap, possibly because of differences in sets of genes screened, cell types, and phenotypes visualized.

An ROS dependant physiological mechanism that triggers proteasomal clearance of VAP(P58S) aggregation

In our study, we have used a dosage-dependent pan-neuronal GAL4 expression of VAP(P58S) in order to study changes in aggregation in the third instar larval brain. We 
which, led to a decrease in VAP(P58S) aggregation accompanied by oxidative stress. We identified a role of ROS in upregulating the proteasomal machinery and, thereby facilitating the degradation of misfolded VAP(P58S) protein/aggregates (Integrated Model; Fig. 8A). However, in absence of ROS, we did not find any change in aggregation density upon pharmacological proteasomal inhibition. This is consistent with the cell culture studies that point towards the downregulation of Ubiquitin-proteasome system (UPS) with VAP(P58S) aggregation as a dominant negative effect on wild type VAP 393 function (KANEKURA et al. 2006; GKOGKAS et al. 2008; PAPIANI et al. 2012; GENEVINI et al. 2014). Overexpression of VAP(P58S) or loss of VAP in Drosophila has been shown to enhance ER stress in the adult brains and may be a result of suspended proteasomal 396 degradation (TSUDA et al. 2008; MoustAQIM-BARRETTE et al. 2014). In mice, VAP(P56S) 397 aggregates have been shown to represent an ER-Quality Control (ERQC) compartment 398 that develops as a result of a debilitated ER-Associated Degradative (ERAD) pathway 399 (KUIJPERS et al. 2013). Indeed, VAP has been shown to interact with UPR sensor AFT6 400 in mice and the ERAD complex thereby regulating proteostasis and lipid homeostasis in 401 HeLa cell lines (Gkogkas et al, 2008; Ernst et al, 2016). Studies in mammalian cell lines 402 suggest that VAP(P56S) is ubiquitinated, aggregates on the ER membrane and is cleared 403 by the AAA+ valsolin containing protein (VCP)/p97, which interacts with Fas associated 404 factor 1(FAF1) and may use the FFAT motif in FAF1 as an adapter to interact with VAP 405 (PAPIANI et al. 2012; BARON et al. 2014). In Drosophila, VAP has been shown to be 406 essential for ER homeostasis by maintaining lipid transport, whereas the mutant VAP flies 407 show accumulation of ubiquitinated and membrane proteins in neuronal cells 408 (MoustaqIM-BARRETTE et al. 2014). Hence, although ER stress is build up with 409 VAP(P58S) aggregation, it does not lead to subsequent oxidative stress, as shown in our 410 results. This suggests that ROS enhances the proteasomal degradation of VAP(P58S) 411 through an ER stress-independent mechanism. Although neuronal VAP(P58S) 412 aggregates appeared to be non-toxic to flies per se, our study highlights the effects of 413 ROS on the dynamics of VAP(P58S) from misfolded protein to aggregate formation and 414 subsequent clearance. 
TOR signaling regulates VAP(P58S) dynamics by a UPS dependent and Atg1 418 independent mechanisms.

We previously identified mTOR pathway as a strong regulator of both VAP and VAP(P58S) phenotypes at the neuromuscular junction (DEIVASIGAMANI et al. 2014). Here, we have shown that inhibition of mTOR pathway also reduces VAP(P58S) aggregation levels in third instar larval brains in presence of ROS. mTOR pathway downregulation is

423 known to activate autophagy (NODA AND OHSUMI 1998), a process that has been shown 424 to reduce mutant huntingtin fragments (RAVIKUMAR et al. 2004) and amyloid- $\beta$ levels 425 (SPILMAN et al. 2010) in mice models. Autophagy has been suggested to be upregulated 426 in presence of VAP(P56S) aggregates that also colocalize with the autophagic marker, 427 p62, in mice (LARROQUETTE et al. 2015). With VAP knockdown in cell culture, autophagy 428 is upregulated due to the loss of calcium homeostasis that arises with the disruption of 429 ER-mitochondrial contact sites (GomEZ-SUAGA et al. 2017a; GomEZ-SuAGA et al. 2017b). 430 However, VAP is also suggested to have a role in autophagosomal biogenesis through 431 direct interaction with autophagy proteins (ZHAO et al. 2018). In our study, we do not 432 observe any clearance of VAP(P58S) aggregation with activation of Atg1, indicating that 433 clearance observed with mTOR inhibition may be an effect of one or more of its downstream processes (Fig. 8A).

mTOR and SOD1 have been shown to be genetic interactors in Drosophila with mTOR inhibition enhancing the lifespan defect incurred with SOD1 knockdown (SUN et al. 2012). Recently, mTOR has been directly shown to regulate SOD1 activity by its phosphorylation based on nutrient availability in yeast and mammalian cells (TSANG et al. 2018). Although this phosphorylation site does not appear to be conserved in Drosophila, this study demonstrates the role of mTOR pathway in regulating ROS via SOD1. mTOR inhibition, specifically, mTORC1 has also been shown to activate proteasomal 442 degradation independent of its other targets, such as, 4EBP, S6K and Ulk (CAVANAUGH 443 et al. 2006; ZHAO et al. 2015). An evolutionarily conserved regulation of components of 444 proteasomal assembly by mTORC1 via Mpk1/ERK5 has been reported in yeast as well 445 as mammalian cell culture (ROUSSEAU AND BERTOLOTTI 2016). ERK5 signalling has been 446 implicated in neuroprotective roles in response to mild levels of oxidative stress 447 (CAVANAUGH et al. 2006; Su et al. 2014). These studies suggest that ROS regulation by 
448 mTOR inhibition via SOD1 and ERK5, serves as a plausible mechanism for the 449 proteasomal degradation of VAP(P58S) protein/aggregation, and by extension, the rescue of VAP(P58S) NMJ phenotype (DEIVASIGAMANI et al. 2014) (Fig. 8B).

Increase in ROS by VAP, but not VAP(P58S) expression plausible factor of motor neuron death in ALS (BARBER et al. 2006; SACCON et al. 2013). Teuling et al., 2007 (TEULING et al. 2007) have shown that VAPB protein levels decrease in an age-dependent manner in a mouse model of SOD1-G93A, providing the first evidence of a link between ALS1 and VAP/ALS8. We now find that overexpressed VAP, unlike VAP(P58S), promotes the accumulation of ROS in the system. This is consistent with a study that shows lowered ROS in a vpr (VAP ortholog) mutant of C. elegans in response to increased mitochondrial connectivity and altered function (HAN et al. 2012). VAP neuronal overexpression in Drosophila has also been shown to increase bouton number (PENNETTA et al. 2002) similar to SOD1 mutant phenotype at the NMJ (MILTON et al. 2011), and is correlated with increased ROS in both scenarios. VAP may be important in regulating pathways that respond to changes in ROS levels, such as mTOR and ERK pathways that can regulate UPS (ROUSSEAU AND BERTOLOTTI 2016). VAP also modulates ERAD (and UPS), via its interaction with VCP and FAF1 (PAPIANI et al. 2012; BARON et al. 2014). We hypothesize that the interaction between VAP and ROS could lead to crosstalk between these pathways regulating global proteostasis (Fig. 8B).

ROS may regulate VAP levels by regulating VAP transcription

In our study, we have found that in presence of ROS, VAP transcription is 472 downregulated in wild type flies. We had previously shown that SOD1 knockdown 473 rescues VAP macrochaetae phenotype (DEIVASIGAMANI et al. 2014), which may be a 474 consequence of excessive ROS accumulation, and subsequent downregulation of VAP 475 levels and function. Two independent studies (QIU et al. 2013; KIM et al. 2016), that 476 overexpressed VAPB in ALS1 (SOD1-G93A) mice as an attempt at rescuing ALS 477 defects, found contradictory observations, owing mainly to differences in expression 478 levels of the protein. VAPB mRNA levels are known to be lowered in spinal cords of 
479 patients with sporadic ALS (ANAGNOSTOU et al. 2010), as well as in IPSC- derived motor 480 neurons from ALS8 patients (MITNE-NETO et al. 2007). It has also been reported that 481 VAPB staining in motor neurons of sporadic patients is increased showing "punctate 482 accumulation" that colocalize with early endosomal marker, Rab5 (SANHUEZA et al. 2015). 483 Based on our results and taking into consideration earlier observations (TEULING et al. 484 2007; AnAgnostou et al. 2010; Deivasigamani et al. 2014), we submit that in the ALS 485 disease scenario, increased VAP accumulates ROS that initiates a negative feedback 486 loop resulting in downregulation of VAP, at the transcript level (Fig. 8A). It remains to be 487 tested whether ROS-activated pathways such as MAP kinase pathways or mTOR 488 pathway, could directly control VAP expression. This VAP/ROS regulation that we have 489 uncovered may have significant implications in ALS pathogenesis for both sporadic and 490 familial ALS.

In Summary, we find that the dynamics of VAP(P58S) neural aggregates, a 493 species intimately linked to disease in the human context, is sensitive to levels of ROS. 494 Change in physiological levels of ROS appear to dictate the equilibrium between the 495 aggregated and non-aggregated forms. The cellular levels of ROS are themselves 496 dictated by well characterized regulatory mechanisms that include ROS generators and 497 scavengers. As shown in this study, TOR signalling and VAPNAP(P58S) expression 498 levels would contribute to the extent of aggregation, and may act as regulatory feedback 499 loops to regulate physiological ROS levels. SOD1, VAPIALS8, TOR and ROS are part of 500 physiological regulatory circuit that maintains levels of VAP(P58S) aggregates. 
Materialls \& Methods

502 Generation of constructs and dsRNA: The cDNA sequence of VAP and VAP(P58S)

503 mutant were cloned into pRM-GFP plasmid (BHASKAR et al. 2000) to generate both $\mathrm{N}$ and

504 C-terminal GFP fusions, using the EcoR1 restriction site. The pRM-GFP vector has GFP 505 cloned into pRM-HA3 vector at the BamHI site. 500 uM CuSO4 was used to drive expression is S2R+ cells after transient transfections. dsRNA for the secondary screen was generated using MEGAscript ${ }^{\circledR}$ T7 Kit (AM1333) by ThermoFisher Scientific.

508 Template for dsRNA was generated by using cDNA as template, prepared from flies. 509 Primers for the same were ordered from Sigma.

510 Handling of Schneider cells: Drosophila S2R+ cells were maintained in Schneider cell 511 Media (\#21720-024; GIBCO) with 10\% Heat inactivated Fetal Bovine Serum (FBS, 512 \#10270; GIBCO). Batches of cells were frozen in 10\% DMSO (D2650; Sigma) and stored 513 in liquid nitrogen following DRSC protocol (http://www.flyrnai.org/DRSC-PRC.html). In 514 general, after reviving, cells were discarded after 25-30 passages. Cells were maintained 515 at $23^{\circ} \mathrm{C}$, and split every 4 days at a ratio of $1: 5$.

516 Cell culture and generation of S2R+ stable lines: Stable S2R+ cell lines were generated 517 by co-transfecting with pRM-HA3 constructs of VAP:GFP, VAP(P58S):GFP or GFP along 518 with pCo-Hygro in 20:1 ratio, using Effectene (QIAGEN) and/or Mirus TransIT 2020 (MIR 5195400 ), and selected under $250 \mu \mathrm{g} / \mathrm{ml}$ of hygromycin (Sigma) for 10-15 passages. Stable 520 as well as transiently transfected cell lines were induced to express the gene of interest 521 under a metallothionein promoter using increasing concentrations $250 \mu \mathrm{M}, 500 \mu \mathrm{M}, 750 \mu \mathrm{M}$ 522 and $1000 \mu \mathrm{M}$ of copper sulphate and analysed at 12, 24, 36 and 48 hours post induction. 523 Transient transfections assays were performed using Mirus TransIT-2020 (MIR 5400) 524 transfection reagent. Protocol for dsRNA knockdown assay was modified from (RoGERS 525 AND RogERS 2008). Fixation, DAPI staining and imaging was done using EVOS FL Auto 526 Cell Imaging system. Super-resolution images of fixed VAP:GFP and VAP(P58S):GFP 527 cells were acquired using Leica SR GSD 3D system.

528 Western blotting: Cells were centrifuged at 3000 rpm for 5 minutes in Eppendorf 5414R 529 centrifuge. The pellet was resuspended in $20 \mu$ of supernatant and boiled with 1 X SDS 530 Dye at $95^{\circ} \mathrm{C}$. Samples were centrifuged again at 10000 rcf for 10 minutes. Cell extracts 531 were separated by 12\% SDS-PAGE and transferred onto $0.45 \mu \mathrm{m}$ PVDF membrane 
532 (Millipore). Membranes were blocked for 1 hour in 5\% skimmed milk in 1X TBS containing $5330.1 \%$ Tween-20 at room temperature and probed with 1:10,000 diluted mouse anti534 Tubulin (T6074; Sigma-Aldrich) and 1:5,000 diluted mouse anti-GFP (Roche life science), 535 overnight at $4{ }^{\circ} \mathrm{C}(12$ hours $)$. Anti-rabbit and anti-mouse secondary antibodies conjugated 536 to horseradish peroxide (Pierce) were used at a dilution of 1:10,000 for 1 hour at room 537 temperature. Blots were developed with Immobilon Chemiluminescent Substrate 538 (LuminataClassico Western HRP substrate from Millipore) using a LAS4000 Fuji imaging 539 System.

540 GO analysis: The list of genes and Gene Ontology (GO) information was obtained based 541 on Flybase (http://flybase.org) (MARYGOLD et al. 2013) entries. Genes were categorized 542 manually in the broad categories of ALS genes, VAP interactome (DEIVASIGAMANI et al. 543 2014) and proteostasis. List of ALS loci and ALS related genes were obtained from 544 http://alsod.iop.kcl.ac.uk/(WROE et al. 2008). The Drosophila melanogaster homologs of 545 these ALS genes were identified using Ensembl biomart tool 546 (http://asia.ensembl.org/biomart/martview) and Flybase batch download tool. Human 547 orthologs of the target genes listed in Suppl. Table 1C and 1D were identified using 548 DRSC Integrative Ortholog Prediction Tool (DIOPT) (http://www.flyrnai.org/cgibin/DRSC orthologs.pl).

550 High through-put screen, and image acquisition: The screen was performed at the 551 screening facility at CCAMP-NCBS, Bangalore (http://ccamp.res.in/HTS-HCI). dsRNA for 552 the high throughput screen was generated and plated into sixteen 384 well plates by 553 Chromous Biotech, Bangalore in preparation for the experiment. The library used as a 554 template for generating dsRNAs was procured from Open Biosystems (RDM1189 and 555 RDM4220). $50 \mu$ of cells $\left(3 \times 10^{\wedge} 6 / \mathrm{ml}\right.$ ) were plated in each well for the 384 well flat 556 bottom plates obtained from Corning. Each target dsRNA knockdown experiment was 557 done in triplicate, randomly arranged in the 384 well plate. The cells were treated with 10 $558 \mu \mathrm{g} / \mathrm{ml}$ of dsRNA for 48 hours, followed by induction with $500 \mu \mathrm{M} \mathrm{CuSO}_{4}$. The cells were 559 fixed and imaged at 24 and 36 hours post $\mathrm{CuSO}_{4}$ induction. Fixation was done with $4 \%$ 560 PFA in 1 X PBS, washed twice with 1 X PBS, treated with $0.05 \mu \mathrm{g} / \mathrm{ml}$ DAPI and followed 561 with two washes with 1X PBS. Each plate contained 7 negative controls occupying 42 562 wells. 114 unique genes were screened in each plate. Few genes were kept as overlap 
563 between multiple plates to check for their consistency and reproducibility. Imaging for the

564 high throughput screen was performed by THERMO Array Scan VTI HCS system. Dual565 channel images from ten fields in each well were captured using a 20X air objective and 566 an EMCCD camera. The FITC (488nm) channel was used for imaging VAP(P58S):GFP 567 aggregates and the DAPI (405nm) channel for imaging cell nuclei. 10 fields were imaged 568 in each well and around 400 cells were imaged per field. In well triplicates, around 12,000 569 cells were imaged for each dsRNA knockdown.

570 High throughput data analysis: Images from the FITC and DAPI channels in each site 571 were read using the Bio-Formats MATLAB toolbox (LINKERT et al. 2010) and were 572 processed using custom MATLAB scripts. The segmentation was done using the DAPI 573 images and the extraction of pixel intensities was done on the FITC channel. Illumination 574 correction was performed as a pre-processing step on the DAPI Images and individual 575 nuclei were segmented after a contrast stretching routine was applied. The identified 576 objects were further filtered for outliers, based on a size-based cutoffs and the individual 577 8-connected components were labelled as separate nuclei. Under 20x magnification we 578 estimated the cellular radius to be around 10 pixels corresponding to $5 \mu \mathrm{m}$. Thus, labelled 579 cellular objects (ROIs), were obtained by dilating the centroids of each nuclei by 10 pixels.

580 Around 400 ROls were obtained from each field consistent with manually counted cells 581 in these images. The resultant ROl's were further filtered for clumps and out of focus 582 objects. The GFP intensities were obtained for these ROl's post a local background 583 correction of the FITC images (with a disk size of 3 pixels). Average and total intensities 584 were calculated from the pixel data obtained from every cell/ROI from these FITC images. 585 A Kolmogorov-Smirnov-like (KS) statistic was used to assign Z-scores to each gene on 586 plate as reported by (DEY et al. 2014). A statistically significant threshold was obtained 587 for the triplicate data using monte-carlo simulations. Genes were classified as hits, if it 588 occurred two or more times above a given Z-score threshold. The false positive rates for 589 both parameters at both time points was zero. The false negative rates for average 590 intensity for 24 hours- time point was 0.2523 and for 36 hours- time point was 0.361 . The 591 false negative rates for total intensity for 24 hours- time point was 0.3838 and for 36 hours592 time point was 0.3164 . 
593 Fly husbandry and brain aggregation assay: Fly lines were maintained on standard corn 594 meal agar medium. UAS-GAL4 system was used for overexpression of transgenes. UAS595 VAP wildtype, UAS-VAP(P58S) and C155-GAL4 lines used for fly experiments have 596 been described earlier (RATNAPARKHI et al. 2008; DeIVASIgAmANI et al. 2014). Canton S 597 flies were used as wildtype control. UAS-VAP_i (27312), UAS-SOD1_i (34616, 29389, 598 36804) and UAS-TOR_i (35578) where the suffix ' $l$ ' indicates an RNAi line, and UAS599 SOD1 (24750, 33605) were obtained from BDSC. Clone for UAS-FLAG-HA tagged 600 SOD1 in pUASt vector was obtained for expression in Drosophila from DGRC and 601 injected in the NCBS-CCAMP transgenic facility. UAS-Atg1 line was kindly provided by 602 Dr. Chen, Academia Sinica; the line was validated in the wing using ptc-GAL4 as 603 described (CHEN et al. 2008). Experimental Crosses were set at $18^{\circ} \mathrm{C}, 25^{\circ} \mathrm{C}$ or $28^{\circ} \mathrm{C}$, as 604 indicated. Brains were dissected from third instar larvae and processed for 605 immunostaining assay. 4\% paraformaldehyde containing $0.1 \%$ Triton-X was used for 606 fixation followed by washes with 1X PBS. Blocking treatment and washes were 607 performed with 0.3\% Triton-X with 2\% BSA. Brains were stained with 1:500 diluted anti608 VAP antibody and 1:1000 anti-rabbit secondary (Invitrogen) was used. Z-stacks of five609 ten brains for each sample were imaged under 63X oil objective of Ziess LSM 710 610 Confocal Microscope. The number of aggregates were quantified per cubic micron of the 611 ventral nerve cord, defined as "aggregation density" using the Huygen professional 612 software. The high intensity puncta were considered as aggregates. An arbitrary 613 threshold was set for controls as well as for test samples that achieved removing low 614 intensity background signal emitted by the tissue, along with separation of high intensity 615 puncta that were adjacent to one another. An object filter was used to remove objects of 616 size greater than 1000 pixels and garbage size smaller than 10 pixels was excluded.

617 Three 3D region of interests of fixed size were drawn along the tip of the ventral nerve 618 cord and the number of aggregates were counted from each of these ROls and averaged 619 for each animal. The volume (in cubic micron) of ROI depicting the thickness of the brain 620 tissue was measured as the range of the z-stack of the image. The aggregation density 621 obtained for each brain has been normalised to the mean of the control group, C155622 GAL4; UAS-VAP(P58S) (+ 0.25\% DMSO, in case of DMSO-soluble drug experiments) 
623 and plotted as "normalized aggregation density" in each graph. Student t-test and one624 way ANOVA were used to measure statistical significance.

625 Drug treatment: Cells were exposed to $10 \mathrm{mM}$ and $20 \mathrm{mM}$ Paraquat dichloride hydrate 626 (500mM, 36541-Sigma-aldrich) for 24 hours prior to protein induction with 500 $4 \mathrm{M}$ copper 627 sulphate. Fixation, DAPI staining and imaging was done using EVOS FL Auto Cell 628 Imaging system. For flies, 10-12 virgins were placed with CS males, for each genotype 629 and animals were allowed to mate for 24 hours and transferred to standard cornmeal fly 630 media containing paraquat $(0.05 \mathrm{mM}, 0.5 \mathrm{mM}$, and $5 \mathrm{mM}), \mathrm{MG} 132(5 \mu \mathrm{M})$, rapamycin 631 (200nM) or DMSO (0.25\%).

632 Oxyblot assay: Third instar larval brains were lysed in RIPA containing 50 mM DTT and 633 centrifuged at 10000 rcf. The lysate containing $10 \mu \mathrm{g}$ of protein was incubated with 2,4634 dinitrophenylhydrazine (DNPH) to derivatize the carbonyl groups of oxidized proteins with 635 2,4-dinitrophenylhydrazone (DNP-hydrazone) as described by the Oxyblot Protein 636 Oxidation Detection Kit (S7150) from EMD Milipore. The derivatized protein lysate was 637 separated on a 12\% SDS-PAGE and transferred onto $0.45 \mu \mathrm{m}$ PVDF membrane 638 (Millipore). Oxidized protein levels in the lysate were detected by probing with anti-DNP 639 antibody on western blot as per the Oxyblot Protein Oxidation Detection Kit manual.

640 Lipid extraction and targeted LC-MS lipidomics: All MS quantitation phospholipid 641 standards were purchased from Avanti Polar Lipids Inc., USA. The brain samples were 642 washed with PBS ( $x 3$ times), and transferred into a glass vial using $1 \mathrm{~mL}$ PBS. $3 \mathrm{~mL}$ of $6432: 1$ ( $\mathrm{vol} / \mathrm{vol}$ ) $\mathrm{CHCl}_{3}$ : $\mathrm{MeOH}$ with the internal standard mix (1 nmol 17:1 FFA, 100 pmol 644 each of 17:0-20:4 PS, 17:0-20:4 PC, 17:0-20:4 PE, and 17:0-20:4 PA) was added, and 645 the mixture was vigorously vortexed. The two phases were separated by centrifugation 646 at $2800 \times \mathrm{g}$ for 5 minutes. The organic phase (bottom) was removed, $50 \mu \mathrm{L}$ of formic acid 647 was added to acidify the aqueous homogenate (to enhance extraction of phospholipids), 648 and $\mathrm{CHCl}_{3}$ was added to make up $4 \mathrm{~mL}$ volume. The mixture was vortexed, and separated 649 using centrifugation described above. Both the organic extracts were pooled, and dried 650 under a stream of $\mathrm{N}_{2}$. The lipidome was re-solubilized in $200 \mu \mathrm{L}$ of $2: 1$ (vol/vol) $\mathrm{CHCl}_{3}$ : $651 \mathrm{MeOH}$, and $20 \mu \mathrm{L}$ was used for the targeted LC-MS analysis (KAMAT et al. 2015). All the 652 phospholipid species analyzed in this study were quantified using the multiple reaction 653 monitoring (MRM) method on an AbSciex QTrap 4500 LC-MS with a Shimadzu Exion-LC 
654

655

656

657

658

659

660

661

662

663

664

665

666

667

668

669

670

671

672

673

674

675

676

677

678

679

680

681

682 series quaternary pump. All data was collected using the Acquisition mode of the Analyst software, and analyzed using the Quantitate mode of the same software. The LC separation was achieved using a Gemini 5U C-18 column (Phenomenex, 5 um, 50 x 4.6 $\mathrm{mm}$ ) coupled to a Gemini guard column (Phenomenex, 4 × $3 \mathrm{~mm}$, Phenomenex security cartridge). The LC solvents were: For positive mode: buffer A: $95: 5$ (vol/vol) $\mathrm{H}_{2} \mathrm{O}: \mathrm{MeOH}$ + 0.1\% formic acid + 10 mM ammonium formate; and buffer B: 60:35:5 (vol/vol) iPrOH: $\mathrm{MeOH}: \mathrm{H}_{2} \mathrm{O}+0.1 \%$ formic acid $+10 \mathrm{mM}$ ammonium formate, For Negative mode: buffer A: $95: 5$ (vol/vol) $\mathrm{H}_{2} \mathrm{O}: \mathrm{MeOH}+0.1 \%$ ammonium hydroxide; and buffer $\mathrm{B}: 60: 35: 5$ (vol/vol) iPrOH: MeOH: $\mathrm{H}_{2} \mathrm{O}+0.1 \%$ ammonium hydroxide. All the MS based lipid estimations was performed using an electrospray ion source, using the following MS parameters: ion source $=$ turbo spray, collision gas $=$ medium, curtain gas $=20 \mathrm{~L} / \mathrm{min}$, ion spray voltage $=$ $4500 \mathrm{~V}$, temperature $=400{ }^{\circ} \mathrm{C}$. A typical LC-run consisted of 55 minutes, with the following solvent run sequence post injection: $0.3 \mathrm{ml} / \mathrm{min} 0 \%$ buffer $B$ for 5 minutes, $0.5 \mathrm{ml} / \mathrm{min} 0 \%$ buffer B for 5 minutes, $0.5 \mathrm{ml} / \mathrm{min}$ linear gradient of buffer B from $0-100 \%$ over 25 minutes, $0.5 \mathrm{ml} / \mathrm{min}$ of $100 \%$ buffer $B$ for 10 minutes, and re-equilibration with $0.5 \mathrm{ml} / \mathrm{min}$ of $0 \%$ buffer $B$ for 10 minutes. A detailed list of all the species targeted in this MRM study, describing the precursor parent ion mass and adduct, the product ion targeted can be found in Suppl. Table 2. All the endogenous lipid species were quantified by measuring the area under the curve in comparison to the respective internal standard, and then normalizing to the number of larval brains. All oxidized phospholipids detected were normalized to the corresponding unoxidized phospholipid internal standard. All the data is represented as mean $\pm \mathrm{s}$. e. $\mathrm{m}$. of 4 biological replicates per genotype.

mRNA isolation, cDNA preparation and qRT PCR: About $1 \mu \mathrm{g}$ of mRNA was isolated from 12-18 third instar larval brains using Direct-zol ${ }^{\text {TM }}$ RNA MicroPrep Kit (R2062) from Zymo Research. The cDNA reaction was carried out using High Capacity cDNA Reverse Transcriptase Kit (4368814) by Applied Biosystems. The qPCR reaction was carried out using KAPA SYBR FAST (KK4602) by Sigma using Replex Mastercycler by Eppendorf. The experiment was carried out in three biological replicates with technical triplicates. 


\section{Acknowledgements}

The S2R+ screen was carried out as a paid service at the NCBS:C-CAMP high throughput screening facility. At NCBS, we thank Dr. Satyajit Mayor for his support; MS Shahab Uddin, Lokavya Kurup and Vandana for technical assistance during the execution of the screen; Kausik Chakraborty, IGIB for advice on the analysis of the screen. We thank Bloomington Drosophila Stock Center (BDSC), Indiana, supported by NIH grant P400D018537, for fly stocks; Drosophila Genome Research Centre (DGRC), Indiana supported by NIH grant 2P400D010949 for vectors and clones; TRiP collection at Harvard Medical School (NIH/NIGMS R01-GM084947) for providing transgenic RNAi fly stocks. We thank IISER Microscopy/Confocal Facility and Dr. Nagaraj Balasubramaniam for access to the EVOS system. Shubham Singh and Shabnam Patil are thanked for technical assistance. This work is funded by a research grant from the Department of Biotechnology, Govt. of India (BT/PR8636/AGR/36/786/2013) and Department of Science and Technology, Science and Engineering Research Board (DST-SERB), Govt. of India (EMR/2014/000367) to GR, a DST-SERB Early Career Research Award in Life Sciences (ECR/2016/001261) to SSK, and a DST-FIST infrastructure development grant to the IISER Pune Biology Department. LP was a UG student at IISER and carried out the S2R+ screen at NCBS. KC and SD are/were graduate students supported by research fellowships from CSIR, Govt. of India. KC is an awardee of the DMM conference travel grant. We thank; Anuradha Ratnaparkhi for discussions and comments on the Manuscript, Richa Rikhy for helpful discussions.

\section{Author Contributions}

GR conceived the project and designed the experiments, with input from KC, LP, SSK and SD. KC and LP performed all the experiments. BR wrote the MATLAB code to analyse the screen. SSK contributed by designing and overseeing experiments related to oxidation of proteins and lipids. GR, KC, LP, BR, SD and SSK analysed the data and wrote the Manuscript. The authors declare no conflict of interest. 


\section{References}

Alpy, F., A. Rousseau, Y. Schwab, F. Legueux, I. Stoll et al., 2013 STARD3 or STARD3NL and VAP form a novel molecular tether between late endosomes and the ER. J Cell Sci 126: 55005512.

Anagnostou, G., M. T. Akbar, P. Paul, C. Angelinetta, T. J. Steiner et al., 2010 Vesicle associated membrane protein B (VAPB) is decreased in ALS spinal cord. Neurobiol Aging 31: 969985.

Andersen, P. M., and A. Al-Chalabi, 2011 Clinical genetics of amyotrophic lateral sclerosis: what do we really know? Nature Reviews Neurology 7: 603-615.

Barber, S. C., R. J. Mead and P. J. Shaw, 2006 Oxidative stress in ALS: a mechanism of neurodegeneration and a therapeutic target. Biochim Biophys Acta 1762: 1051-1067.

Baron, Y., P. G. Pedrioli, K. Tyagi, C. Johnson, N. T. Wood et al., 2014 VAPB/ALS8 interacts with FFAT-like proteins including the p97 cofactor FAF1 and the ASNA1 ATPase. BMC Biol 12: 39.

Bhaskar, V., S. A. Valentine and A. J. Courey, 2000 A functional interaction between dorsal and components of the Smt3 conjugation machinery. J Biol Chem 275: 4033-4040.

Castello, P. R., D. A. Drechsel and M. Patel, 2007 Mitochondria are a major source of paraquatinduced reactive oxygen species production in the brain. J Biol Chem 282: 14186-14193.

Cavanaugh, J. E., J. D. Jaumotte, J. M. Lakoski and M. J. Zigmond, 2006 Neuroprotective role of ERK1/2 and ERK5 in a dopaminergic cell line under basal conditions and in response to oxidative stress. J Neurosci Res 84: 1367-1375.

Chai, A., J. Withers, Y. H. Koh, K. Parry, H. Bao et al., 2008 hVAPB, the causative gene of a heterogeneous group of motor neuron diseases in humans, is functionally interchangeable with its Drosophila homologue DVAP-33A at the neuromuscular junction. Hum Mol Genet 17: 266-280.

Chen, G. C., J. Y. Lee, H. W. Tang, J. Debnath, S. M. Thomas et al., 2008 Genetic interactions between Drosophila melanogaster Atg1 and paxillin reveal a role for paxillin in autophagosome formation. Autophagy 4: 37-45.

Cleveland, D. W., and J. D. Rothstein, 2001 From charcot to lou gehrig: deciphering selective motor neuron death in als. Nature Reviews Neuroscience 2: 806-819.

Cluskey, S., and D. B. Ramsden, 2001 Mechanisms of neurodegeneration in amyotrophic lateral sclerosis. Mol Pathol 54: 386-392.

Cocheme, H. M., C. Quin, S. J. McQuaker, F. Cabreiro, A. Logan et al., 2011 Measurement of $\mathrm{H} 2 \mathrm{O} 2$ within living Drosophila during aging using a ratiometric mass spectrometry probe targeted to the mitochondrial matrix. Cell Metab 13: 340-350.

De Vos, K. J., G. M. Morotz, R. Stoica, E. L. Tudor, K. F. Lau et al., 2012 VAPB interacts with the mitochondrial protein PTPIP51 to regulate calcium homeostasis. Hum Mol Genet 21: 1299-1311.

Deivasigamani, S., H. K. Verma, R. Ueda, A. Ratnaparkhi and G. S. Ratnaparkhi, 2014 A genetic screen identifies Tor as an interactor of VAPB in a Drosophila model of amyotrophic lateral sclerosis. Biology Open 3: 1127-1138.

Deng, H. X., A. Hentati, J. A. Tainer, Z. Iqbal, A. Cayabyab et al., 1993 Amyotrophic lateral sclerosis and structural defects in Cu,Zn superoxide dismutase. Science 261: 1047-1051. 
Dey, G., G. D. Gupta, B. Ramalingam, M. Sathe, S. Mayor et al., 2014 Exploiting Cell-To-Cell Variability To Detect Cellular Perturbations. PLoS ONE 9: e90540.

Drechsel, D. A., and M. Patel, 2008 Role of reactive oxygen species in the neurotoxicity of environmental agents implicated in Parkinson's disease. Free Radic Biol Med 44: 18731886.

Echeverri, C. J., and N. Perrimon, 2006 High-throughput RNAi screening in cultured cells: a user's guide. Nat Rev Genet 7: 373-384.

Genevini, P., G. Papiani, A. Ruggiano, L. Cantoni, F. Navone et al., 2014 Amyotrophic lateral sclerosis-linked mutant VAPB inclusions do not interfere with protein degradation pathways or intracellular transport in a cultured cell model. PLoS One 9: e113416.

Gkogkas, C., S. Middleton, A. M. Kremer, C. Wardrope, M. Hannah et al., 2008 VAPB interacts with and modulates the activity of ATF6. Human Molecular Genetics 17: 1517-1526.

Gomez-Suaga, P., S. Paillusson and C. C. J. Miller, 2017a ER-mitochondria signaling regulates autophagy. Autophagy 13: 1250-1251.

Gomez-Suaga, P., S. Paillusson, R. Stoica, W. Noble, D. P. Hanger et al., 2017b The ERMitochondria Tethering Complex VAPB-PTPIP51 Regulates Autophagy. Curr Biol 27: $371-$ 385.

Han, S. M., H. Tsuda, Y. Yang, J. Vibbert, P. Cottee et al., 2012 Secreted VAPB/ALS8 major sperm protein domains modulate mitochondrial localization and morphology via growth cone guidance receptors. Dev Cell 22: 348-362.

Heitman, J., N. R. Movva and M. N. Hall, 1991 Targets for cell cycle arrest by the immunosuppressant rapamycin in yeast. Science 253: 905-909.

Huttlin, E. L., L. Ting, R. J. Bruckner, F. Gebreab, M. P. Gygi et al., 2015 The BioPlex Network: A Systematic Exploration of the Human Interactome. Cell 162: 425-440.

Kamat, S. S., K. Camara, W. H. Parsons, D. H. Chen, M. M. Dix et al., 2015 Immunomodulatory lysophosphatidylserines are regulated by ABHD16A and ABHD12 interplay. Nat Chem Biol 11: 164-171.

Kanekura, K., I. Nishimoto, S. Aiso and M. Matsuoka, 2006 Characterization of amyotrophic lateral sclerosis-linked P56S mutation of vesicle-associated membrane proteinassociated protein B (VAPB/ALS8). J Biol Chem 281: 30223-30233.

Kim, J. Y., A. Jang, R. Reddy, W. H. Yoon and J. L. Jankowsky, 2016 Neuronal overexpression of human VAPB slows motor impairment and neuromuscular denervation in a mouse model of ALS. Hum Mol Genet 25: 4661-4673.

Kory, N., S. Grond, S. S. Kamat, Z. Li, N. Krahmer et al., 2017 Mice lacking lipid dropletassociated hydrolase, a gene linked to human prostate cancer, have normal cholesterol ester metabolism. J Lipid Res 58: 226-235.

Kuijpers, M., V. van Dis, E. D. Haasdijk, M. Harterink, K. Vocking et al., 2013 Amyotrophic lateral sclerosis (ALS)-associated VAPB-P56S inclusions represent an ER quality control compartment. Acta Neuropathol Commun 1: 24.

Larroquette, F., L. Seto, P. L. Gaub, B. Kamal, D. Wallis et al., 2015 Vapb/Amyotrophic lateral sclerosis 8 knock-in mice display slowly progressive motor behavior defects accompanying ER stress and autophagic response. Hum Mol Genet 24: 6515-6529.

Lev, S., D. Ben Halevy, D. Peretti and N. Dahan, 2008 The VAP protein family: from cellular functions to motor neuron disease. Trends Cell Biol 18: 282-290. 
Linkert, M., C. T. Rueden, C. Allan, J. M. Burel, W. Moore et al., 2010 Metadata matters: access to image data in the real world. J Cell Biol 189: 777-782.

Loewen, C. J., A. Roy and T. P. Levine, 2003 A conserved ER targeting motif in three families of lipid binding proteins and in Opi1p binds VAP. EMBO J 22: 2025-2035.

Mandrioli, J., R. D'Amico, E. Zucchi, A. Gessani, N. Fini et al., 2018 Rapamycin treatment for amyotrophic lateral sclerosis: Protocol for a phase II randomized, double-blind, placebocontrolled, multicenter, clinical trial (RAP-ALS trial). Medicine (Baltimore) 97: e11119.

Marygold, S. J., P. C. Leyland, R. L. Seal, J. L. Goodman, J. Thurmond et al., 2013 FlyBase: improvements to the bibliography. Nucleic Acids Res 41: D751-757.

Metz, J., I. G. Castro and M. Schrader, 2017 Peroxisome Motility Measurement and Quantification Assay. Bio Protoc 7.

Milton, V. J., H. E. Jarrett, K. Gowers, S. Chalak, L. Briggs et al., 2011 Oxidative stress induces overgrowth of the Drosophila neuromuscular junction. Proc Natl Acad Sci U S A 108: 17521-17526.

Mitne-Neto, M., C. R. Ramos, D. C. Pimenta, J. S. Luz, A. L. Nishimura et al., 2007 A mutation in human VAP-B--MSP domain, present in ALS patients, affects the interaction with other cellular proteins. Protein Expr Purif 55: 139-146.

Moujalled, D., A. Grubman, K. Acevedo, S. Yang, Y. D. Ke et al., 2017 TDP-43 mutations causing amyotrophic lateral sclerosis are associated with altered expression of RNA-binding protein hnRNP K and affect the Nrf2 antioxidant pathway. Hum Mol Genet 26: 17321746.

Moustaqim-Barrette, A., Y. Q. Lin, S. Pradhan, G. G. Neely, H. J. Bellen et al., 2014 The amyotrophic lateral sclerosis 8 protein, VAP, is required for ER protein quality control. Hum Mol Genet 23: 1975-1989.

Mulligan, V. K., and A. Chakrabartty, 2013 Protein misfolding in the late-onset neurodegenerative diseases: Common themes and the unique case of amyotrophic lateral sclerosis. Proteins 81: 1285-1303.

Murphy, S. E., and T. P. Levine, 2016 VAP, a Versatile Access Point for the Endoplasmic Reticulum: Review and analysis of FFAT-like motifs in the VAPome. Biochim Biophys Acta 1861: 952-961.

Nishimura, A. L., M. Mitne-Neto, H. C. A. Silva, A. Richieri-Costa, S. Middleton et al., 2004 A Mutation in the Vesicle-Trafficking Protein VAPB Causes Late-Onset Spinal Muscular Atrophy and Amyotrophic Lateral Sclerosis. The American Journal of Human Genetics 75: 822-831.

Noda, T., and Y. Ohsumi, 1998 Tor, a phosphatidylinositol kinase homologue, controls autophagy in yeast. J Biol Chem 273: 3963-3966.

Ogrodnik, M., H. Salmonowicz, R. Brown, J. Turkowska, W. Sredniawa et al., 2014 Dynamic JUNQ inclusion bodies are asymmetrically inherited in mammalian cell lines through the asymmetric partitioning of vimentin. Proc Natl Acad Sci U S A 111: 8049-8054.

Paillusson, S., P. Gomez-Suaga, R. Stoica, D. Little, P. Gissen et al., 2017 alpha-Synuclein binds to the ER-mitochondria tethering protein VAPB to disrupt $\mathrm{Ca}(2+)$ homeostasis and mitochondrial ATP production. Acta Neuropathol 134: 129-149. 
Papiani, G., A. Ruggiano, M. Fossati, A. Raimondi, G. Bertoni et al., 2012 Restructured endoplasmic reticulum generated by mutant amyotrophic lateral sclerosis-linked VAPB is cleared by the proteasome. J Cell Sci 125: 3601-3611.

Pennetta, G., P. R. Hiesinger, R. Fabian-Fine, I. A. Meinertzhagen and H. J. Bellen, 2002 Drosophila VAP-33A directs bouton formation at neuromuscular junctions in a dosagedependent manner. Neuron 35: 291-306.

Perluigi, M., F. Di Domenico and D. A. Butterfield, 2015 mTOR signaling in aging and neurodegeneration: At the crossroad between metabolism dysfunction and impairment of autophagy. Neurobiol Dis 84: 39-49.

Prause, J., A. Goswami, I. Katona, A. Roos, M. Schnizler et al., 2013 Altered localization, abnormal modification and loss of function of Sigma receptor-1 in amyotrophic lateral sclerosis. Hum Mol Genet 22: 1581-1600.

Qiu, L., T. Qiao, M. Beers, W. Tan, H. Wang et al., 2013 Widespread aggregation of mutant VAPB associated with ALS does not cause motor neuron degeneration or modulate mutant SOD1 aggregation and toxicity in mice. Mol Neurodegener 8: 1.

Ratnaparkhi, A., G. M. Lawless, F. E. Schweizer, P. Golshani and G. R. Jackson, 2008 A Drosophila Model of ALS: Human ALS-Associated Mutation in VAP33A Suggests a Dominant Negative Mechanism. PLoS ONE 3: e2334.

Ravikumar, B., C. Vacher, Z. Berger, J. E. Davies, S. Luo et al., 2004 Inhibition of mTOR induces autophagy and reduces toxicity of polyglutamine expansions in fly and mouse models of Huntington disease. Nat Genet 36: 585-595.

Rogers, S. L., and G. C. Rogers, 2008 Culture of Drosophila S2 cells and their use for RNAimediated loss-of-function studies and immunofluorescence microscopy. Nat Protoc 3: 606-611.

Rosen, D. R., T. Siddique, D. Patterson, D. A. Figlewicz, P. Sapp et al., 1993 Mutations in Cu/Zn superoxide dismutase gene are associated with familial amyotrophic lateral sclerosis. Nature 362: 59-62.

Rousseau, A., and A. Bertolotti, 2016 An evolutionarily conserved pathway controls proteasome homeostasis. Nature 536: 184-189.

Saccon, R. A., R. K. Bunton-Stasyshyn, E. M. Fisher and P. Fratta, 2013 Is SOD1 loss of function involved in amyotrophic lateral sclerosis? Brain 136: 2342-2358.

Sanhueza, M., A. Chai, C. Smith, B. A. McCray, T. I. Simpson et al., 2015 Network Analyses Reveal Novel Aspects of ALS Pathogenesis. PLoS Genet 11: e1005107.

Spilman, P., N. Podlutskaya, M. J. Hart, J. Debnath, O. Gorostiza et al., 2010 Inhibition of mTOR by rapamycin abolishes cognitive deficits and reduces amyloid-beta levels in a mouse model of Alzheimer's disease. PLoS One 5: e9979.

Stoica, R., K. J. De Vos, S. Paillusson, S. Mueller, R. M. Sancho et al., 2014 ER-mitochondria associations are regulated by the VAPB-PTPIP51 interaction and are disrupted by ALS/FTD-associated TDP-43. Nat Commun 5: 3996.

Stoica, R., S. Paillusson, P. Gomez-Suaga, J. C. Mitchell, D. H. Lau et al., 2016 ALS/FTD-associated FUS activates GSK-3beta to disrupt the VAPB-PTPIP51 interaction and ER-mitochondria associations. EMBO Rep 17: 1326-1342. 
Su, C., F. Sun, R. L. Cunningham, N. Rybalchenko and M. Singh, 2014 ERK5/KLF4 signaling as a common mediator of the neuroprotective effects of both nerve growth factor and hydrogen peroxide preconditioning. Age (Dordr) 36: 9685.

Sun, X., T. Komatsu, J. Lim, M. Laslo, J. Yolitz et al., 2012 Nutrient-dependent requirement for SOD1 in lifespan extension by protein restriction in Drosophila melanogaster. Aging Cell 11: 783-793.

Tarasiuk, J., A. Kułakowska, W. Drozdowski, J. Kornhuber and P. Lewczuk, 2012 CSF markers in amyotrophic lateral sclerosis. Journal of Neural Transmission 119: 747-757.

Taylor, J. P., R. H. Brown, Jr. and D. W. Cleveland, 2016 Decoding ALS: from genes to mechanism. Nature 539: 197-206.

Teuling, E., S. Ahmed, E. Haasdijk, J. Demmers, M. O. Steinmetz et al., 2007 Motor Neuron Disease-Associated Mutant Vesicle-Associated Membrane Protein-Associated Protein (VAP) B Recruits Wild-Type VAPs into Endoplasmic Reticulum-Derived Tubular Aggregates. Journal of Neuroscience 27: 9801-9815.

Tsang, C. K., M. Chen, X. Cheng, Y. Qi, Y. Chen et al., 2018 SOD1 Phosphorylation by mTORC1 Couples Nutrient Sensing and Redox Regulation. Mol Cell 70: 502-515 e508.

Tsuda, H., S. M. Han, Y. Yang, C. Tong, Y. Q. Lin et al., 2008 The Amyotrophic Lateral Sclerosis 8 Protein VAPB Is Cleaved, Secreted, and Acts as a Ligand for Eph Receptors. Cell 133: 963977.

Tudor, E. L., C. M. Galtrey, M. S. Perkinton, K. F. Lau, K. J. De Vos et al., 2010 Amyotrophic lateral sclerosis mutant vesicle-associated membrane protein-associated protein-B transgenic mice develop TAR-DNA-binding protein-43 pathology. Neuroscience 167: 774-785.

Turner, B. J., D. Baumer, N. J. Parkinson, J. Scaber, O. Ansorge et al., 2008 TDP-43 expression in mouse models of amyotrophic lateral sclerosis and spinal muscular atrophy. BMC Neurosci 9: 104.

Turner, M. R., O. Hardiman, M. Benatar, B. R. Brooks, A. Chio et al., 2013 Controversies and priorities in amyotrophic lateral sclerosis. The Lancet Neurology 12: 310-322.

Tyurina, Y. Y., A. A. Shvedova, K. Kawai, V. A. Tyurin, C. Kommineni et al., 2000 Phospholipid signaling in apoptosis: peroxidation and externalization of phosphatidylserine. Toxicology 148: 93-101.

van Blitterswijk, M., M. A. van Es, E. A. Hennekam, D. Dooijes, W. van Rheenen et al., 2012 Evidence for an oligogenic basis of amyotrophic lateral sclerosis. Hum Mol Genet 21: 3776-3784.

Walker, A. K., and J. D. Atkin, 2011 Stress signaling from the endoplasmic reticulum: A central player in the pathogenesis of amyotrophic lateral sclerosis. IUBMB Life: n/a-n/a.

Wroe, R., A. Wai-Ling Butler, P. M. Andersen, J. F. Powell and A. Al-Chalabi, 2008 ALSOD: the Amyotrophic Lateral Sclerosis Online Database. Amyotroph Lateral Scler 9: 249-250.

Yadav, S., R. Thakur, P. Georgiev, S. Deivasigamani, H. Krishnan et al., 2018 RDGBalpha localization and function at membrane contact sites is regulated by FFAT-VAP interactions. J Cell Sci 131.

Zhan, L., Q. Xie and R. S. Tibbetts, 2015 Opposing roles of p38 and JNK in a Drosophila model of TDP-43 proteinopathy reveal oxidative stress and innate immunity as pathogenic components of neurodegeneration. Hum Mol Genet 24: 757-772. 
Zhang, S., R. Binari, R. Zhou and N. Perrimon, 2010 A Genomewide RNA Interference Screen for Modifiers of Aggregates Formation by Mutant Huntingtin in Drosophila. Genetics 184: 1165-1179.

Zhao, J., B. Zhai, S. P. Gygi and A. L. Goldberg, 2015 mTOR inhibition activates overall protein degradation by the ubiquitin proteasome system as well as by autophagy. Proc Natl Acad Sci U S A 112: 15790-15797.

Zhao, Y. G., N. Liu, G. Miao, Y. Chen, H. Zhao et al., 2018 The ER Contact Proteins VAPA/B Interact with Multiple Autophagy Proteins to Modulate Autophagosome Biogenesis. Curr Biol. 


\section{Supplementary Material}

1001 Suppl. Figures along with their legends are part of the Main Manuscript. Suppl. Tables 1002 (described below) have been uploaded formally as 'Suppl. Files'.

1003

1004 Table 1 (Suppl. Table1.xls)

1005 A. List of 900 genes utilized for the screen. List is sorted alphabetically based on gene 1006 symbol.

1007 B. 900 genes, utilized for the screen, classified and listed into 10 categories associated 1008 with ALS or VAP or proteostasis.

1009 C. List of 150 modifiers of VAP(P58S) aggregation, based on average cell intensity, along 1010 with their human orthologs.

1011 D. List of 85 modifiers of VAP(P58S) aggregation, based on total cell intensity, along with 1012 their human orthologs.

1013

1014 Table 2 (Suppl. Table2.xls)

1015 A. Details of the MRM transitions for the different phospholipids measured

1016 B. LC-MS quantitation of the different phospholipids for different genotypes and paraquat 1017 treatment.

1018 C. LC-MS quantitation of the different phospholipids for knockdown of TOR. 


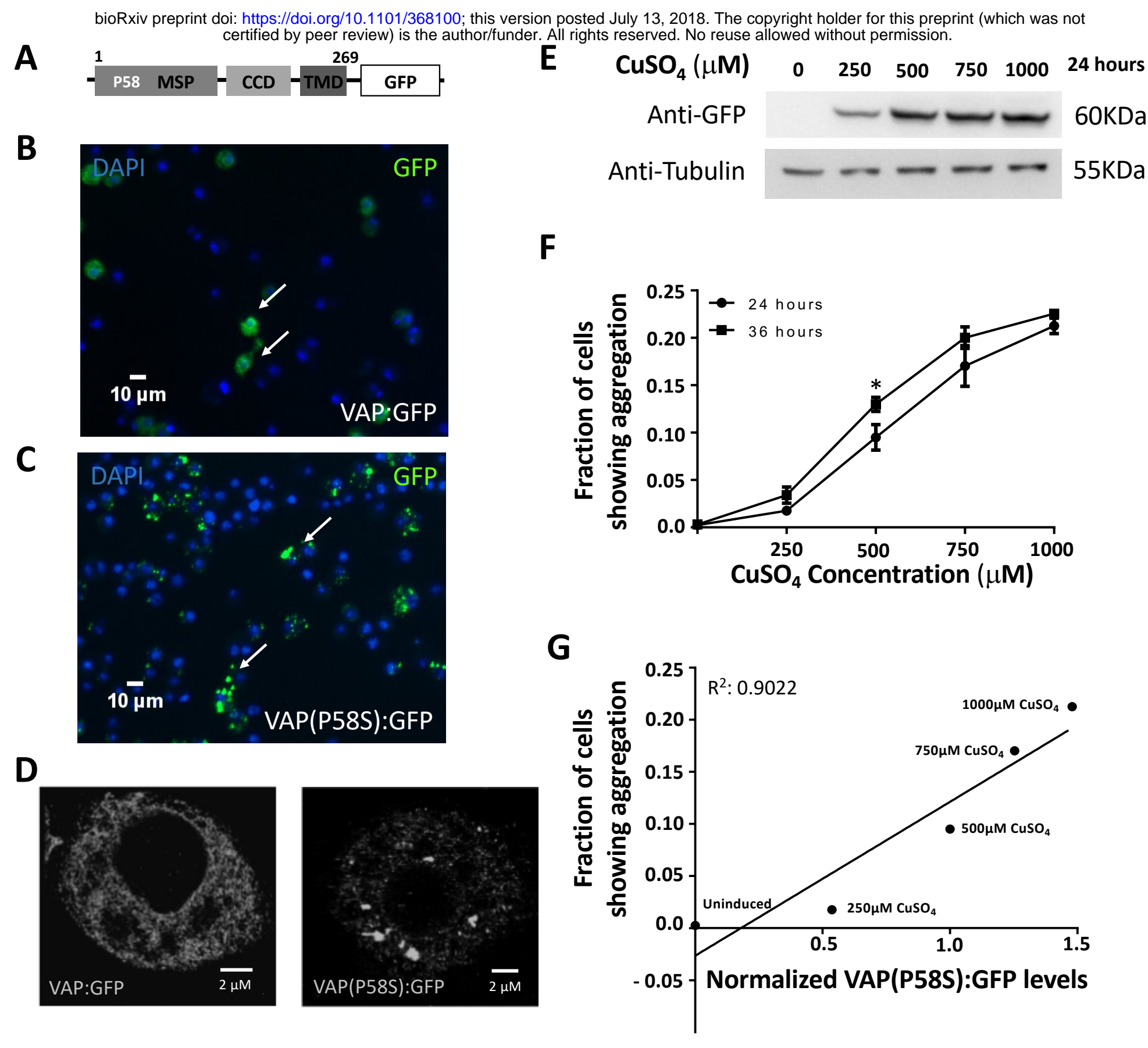

Figure 1: A Drosophila cell culture model to study VAP(P58S) aggregation

A. VAP:GFP and VAP(P58S):GFP when expressed in S2R+ cells allow efficient visualization of VAP protein in the cell by epifluorescence.

B, C. Stable cell lines: expressing $V A P(P 58 S): G F P$, under an inducible metallothionein promoter, results in aggregation (C), unlike VAP:GFP wild-type (B). GFP is visualized by epifluorescence and chromatin by DAPI, postfixation.

D. A super resolution image, using Ground State Depletion microscopy, showing GFP inclusions forming in cells expressing VAP(P58S):GFP but not in VAP:GFP.

E. VAP(P58S):GFP protein levels in cells increase with increasing $\mathrm{CuSO}_{4}$ concentration at 24 hours post induction. F. Increase in fraction of cells showing GFP positive inclusions increases with $\mathrm{CuSO}_{4}$ concentration. At $500 \mu \mathrm{M}$ $\mathrm{CuSO}_{4}$, inclusions significantly increase between 24 hours and 36 hours. Student's t-test (P-value: $\left.{ }^{*}<0.01\right)$ G. A linear correlation between fraction of cells showing aggregation, measured using microscopy plotted against relative VAP(P58S):GFP protein levels, as quantified by western blotting, at 24 hours post induction. 
A bioRxiv preprint doi: https://doALUS/10.ALS/ reatated this version posted July
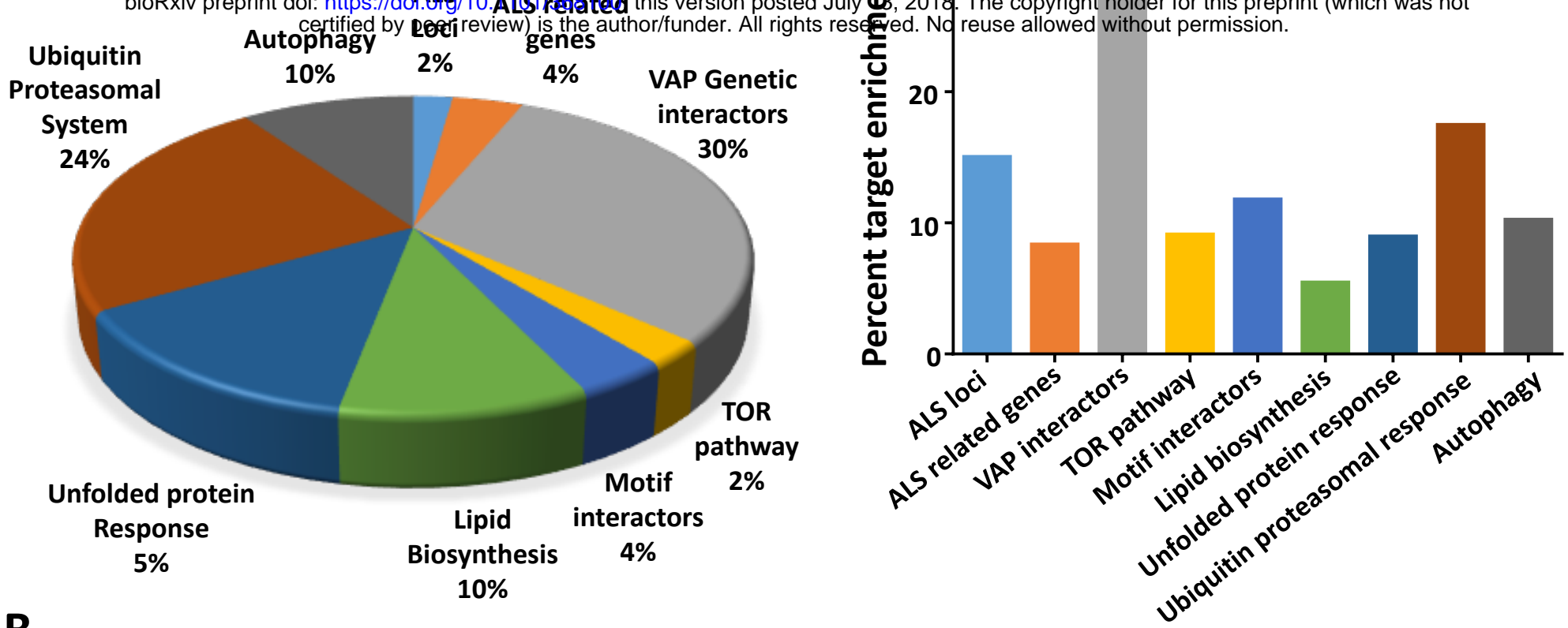

B

1. Raw data import

- Importing GFP and DAPI images (LOCl; BioFormats plugin)

- Plate configuration

- Defining a aggregate for cross-correlation.
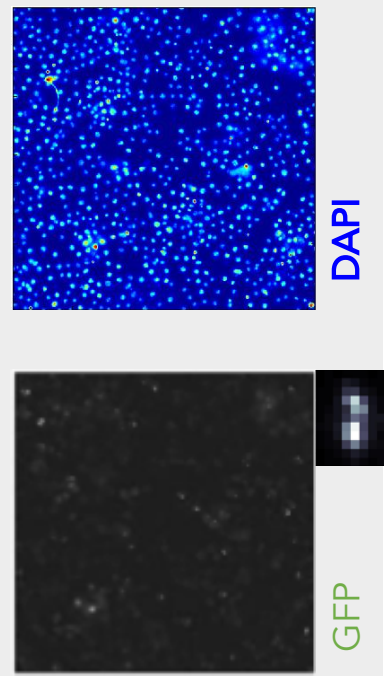

\section{Cell identification}

- Segmentation on DAPI.

- K-means clustering with 2 populations.

- Dilation to generate ROI : 10 points

- Cross-correlation (CC) of aggregate on GFP image
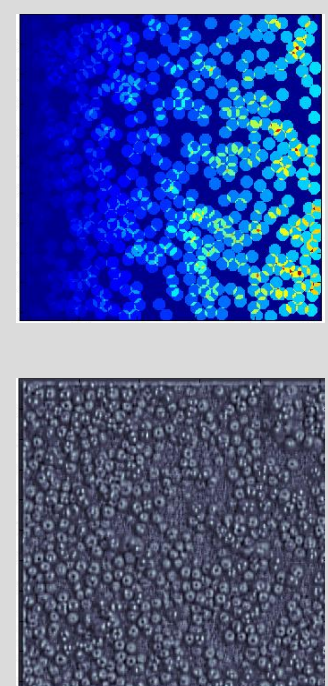

\section{Quality control}

- $30 \%$ ROI overlap for clump deletion.

- Area cut-offs (60pxs) for deletion of illuminated debris.

- CC image filtered for correlation > 0.6
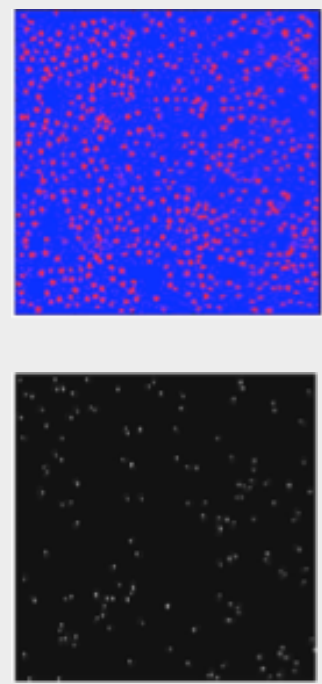

\section{Aggregates}

- Thresholding on ROIs

- K means clustering with 3 populations on GFP image :

- Background

- Foreground

- Aggregate
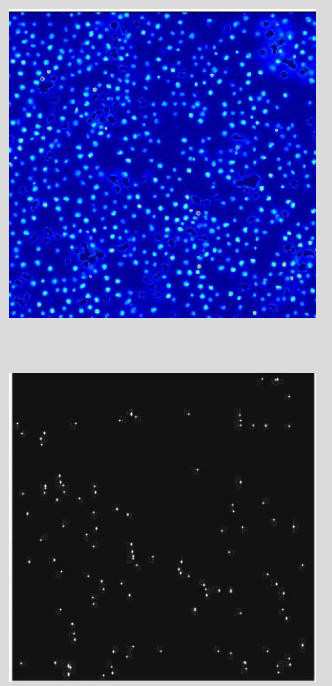

Figure 2: A targeted dsRNA screen in S2R+ cells to discover modifiers of VAP(P58S):GFP aggregation.

A: dsRNA for 900 genes (Suppl. Table 1A) were chosen for knockdown. GO representation indicates the categories of genes chosen and fraction (\%) for each category. Genes were categorized as described in text (Supplementary table $1 A, 1 B)$.

B: Workflow of the steps executed for image analysis using an automated MATLAB script (Dey et al, 2014). Steps detailed in Material and Methods.

C: The end result of the screen is a list of 150 genes identified based on average cell intensity, which have been found to modify aggregation of VAP(P58S):GFP. Graph indicates the percent fold enrichment of targets within each gene category. Genes are listed in Supplementary table 1C. 
bioRxiv preprint doi: https://doi.org/10.1101/368100; this version posted July 13, 2018. The copyright holder for this preprint (which was not certified by peer review) is the author/funder. All rights reserved. No reuse allowed without permission.

A

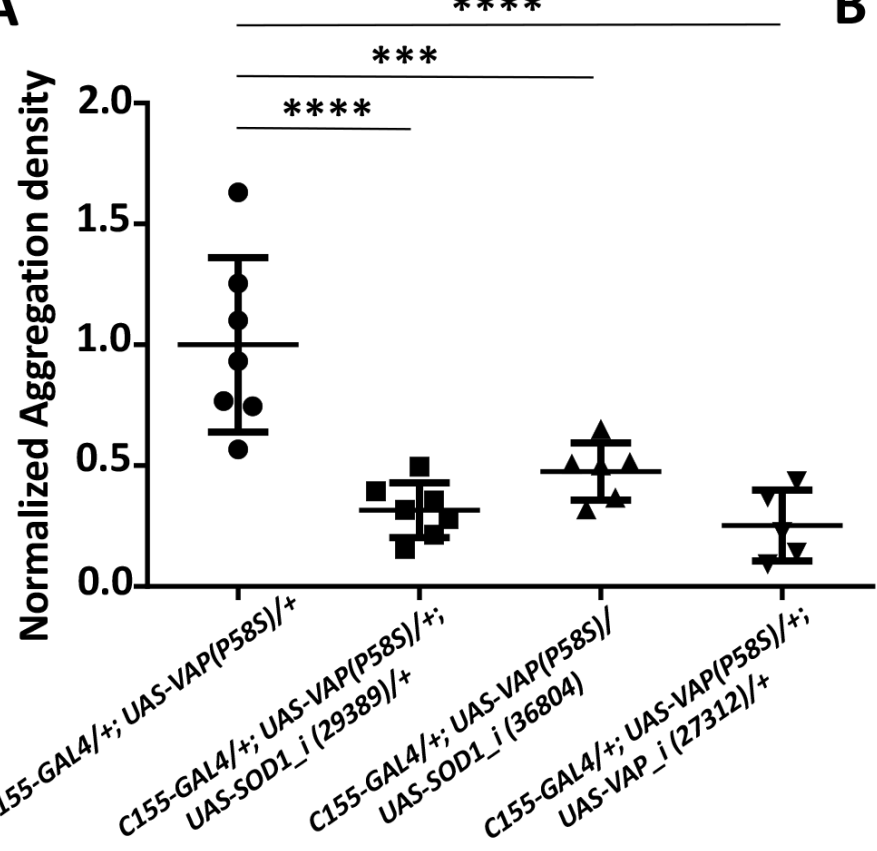

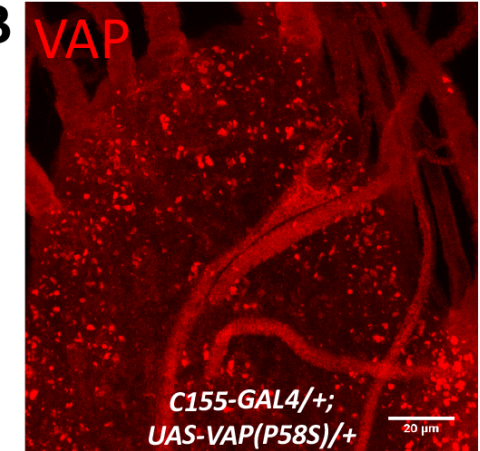

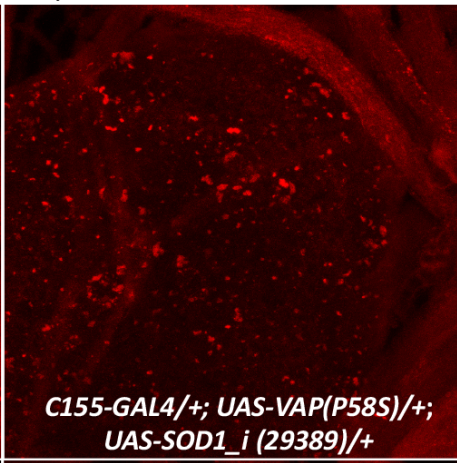

C155-GAL4/+; UAS-VAP(P58S)/ UAS-SOD1_i(36804)
C155-GAL4/+; UAS-VAP(P58S)/+; UAS-VAP_i (27312)/+
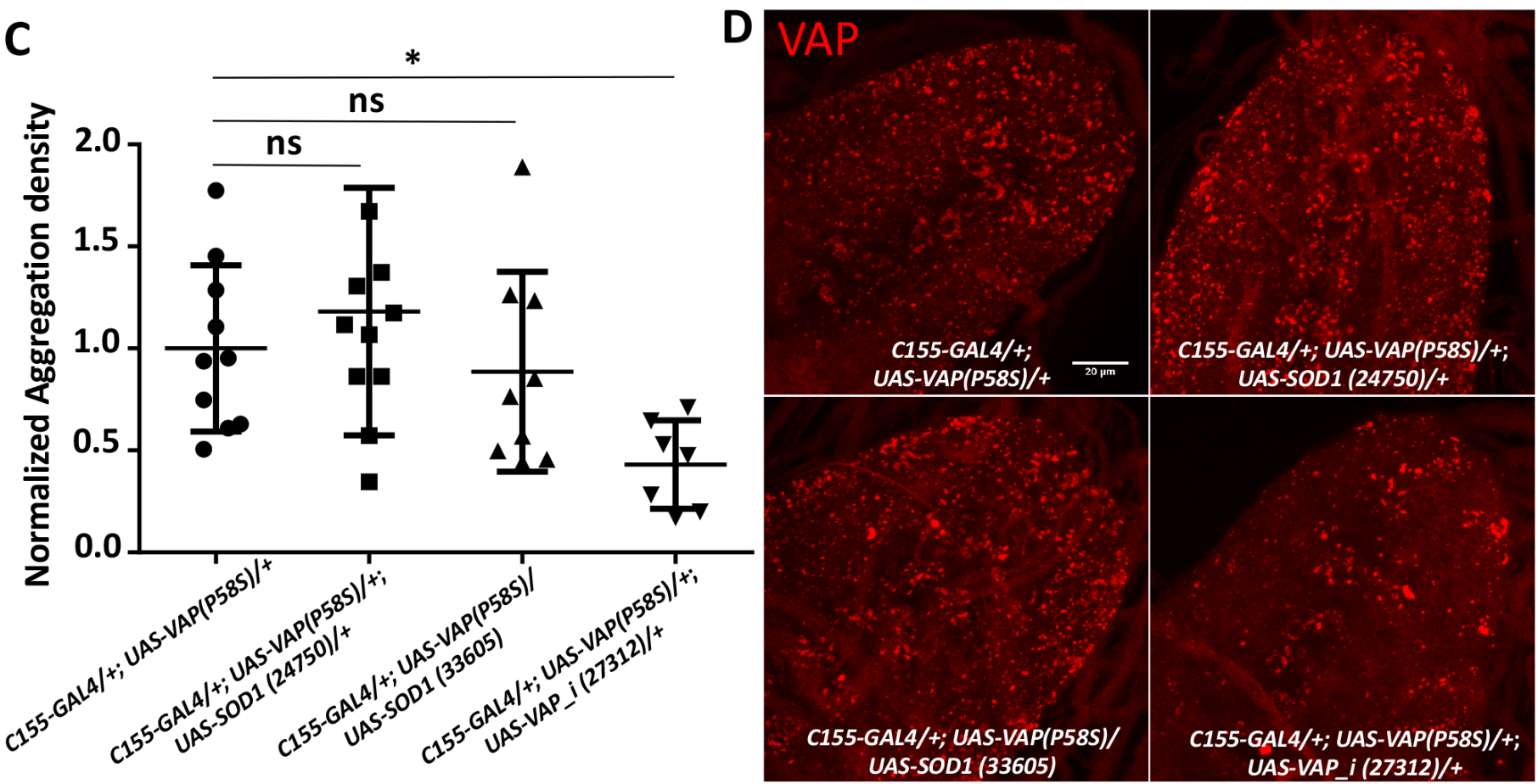

Figure 3: SOD1 loss-of-function reduces VAP(P58S) aggregation in larval brains

A: SOD1 knockdown in the nervous system decreases aggregation density in the ventral nerve cord. VAP knockdown also reduces aggregation due to reduction in VAP protein expression. The ' $i$ ' appended to the gene name indicates an RNAi line. ANOVA ( $P$ value: $* * * *<0.0001)$. Numbers in brackets indicate BDSC stock numbers.

B: Representative images of the ventral nerve cord showing aggregation of VAP(P58S) with SOD1 knockdown(29389 and 36804) and with VAP knockdown (27312).

C: SOD1 overexpression does not affect aggregation density in the ventral nerve cord. ANOVA (P value: *, 0.0208)

D: Representative images of the ventral nerve cord showing aggregation of VAP(P58S) with SOD1 overexpression (24750 and 33605) and with VAP knockdown (27312).

All images were taken at the same magnification. Fisher's LSD multiple comparison (P-values, $*<0.05, * *<0.01$, $* * *<0.001)$. 


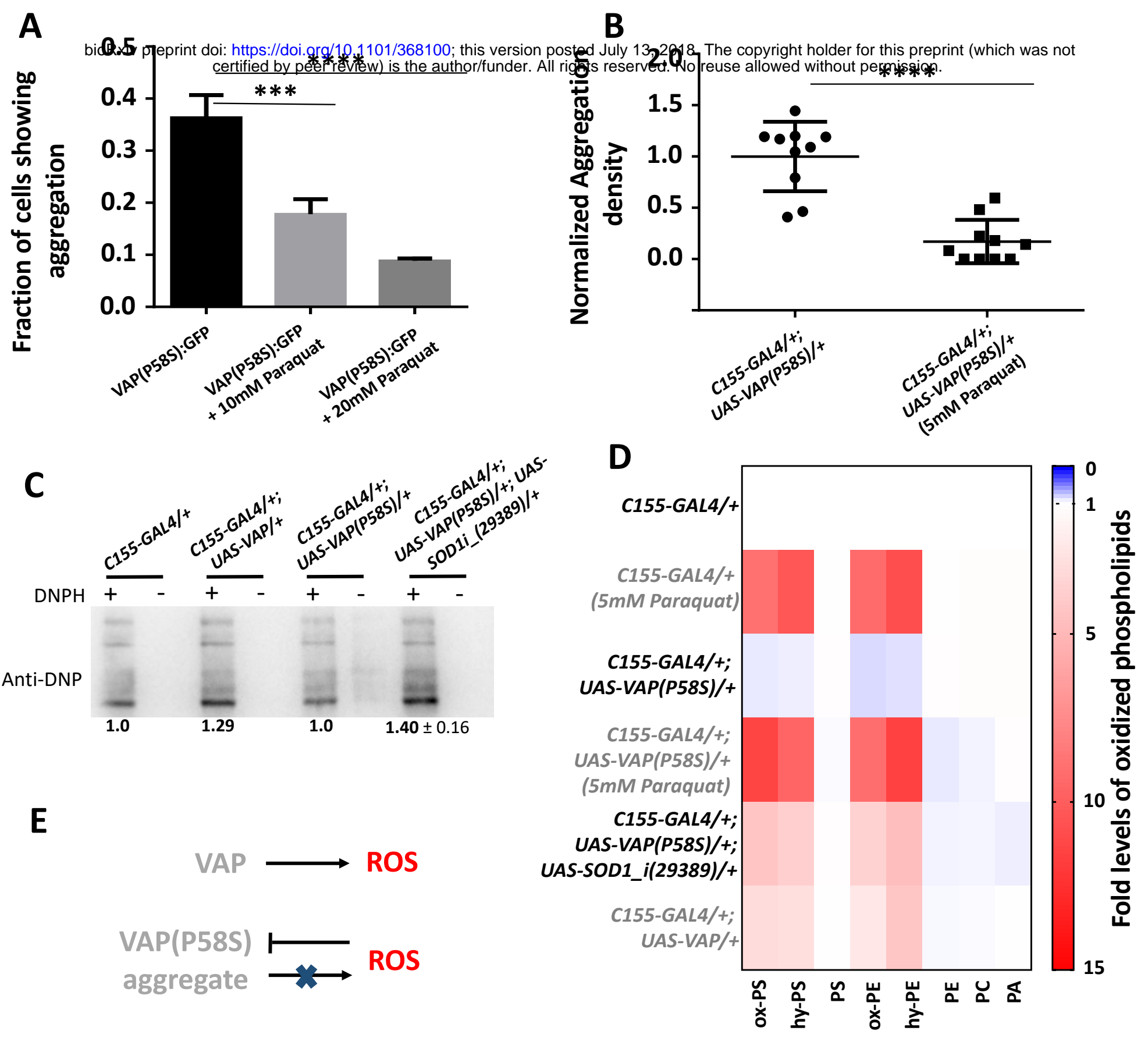

Figure 4: Increase in ROS leads to decrease in VAP(P58S) aggregation levels.

A: 4 hour Paraquat treatment prior to inducing VAP(P58S):GFP in stable S2R+ cell line, reduces the fraction of cells showing aggregation observed 24 hours post-induction. ANOVA (P-value: $* * * *<0.0001)$ Fisher's LSD multiple comparison test (P-values, $* * *<0.001, * * * *<0.0001)$.

B: Paraquat feeding decreases aggregation density in the ventral nerve cord of third instar larval brains in C155-GAL4/+; UAS-VAP(P58S)/+ flies. Student's t-test (P-value: $* * * *<0.0001)$.

C: Oxyblot showing increased levels of oxidized proteins in larval brains $(\mathrm{N}=14)$ upon SOD knockdown, or VAP overexpression. Values below the gel indicate fold intensity of the strongest band, when compared to control (C155-GAL4/+). Suppl. Fig. 4C shows a calibration for the Oxyblot system, values measured after feeding increasing amounts of Paraquat to larvae.

D: Heat map depicting change in levels of oxidized phospholipids normalized to C155-GAL4/+, quantified using MS in response to ROS generated in third instar larval brains $(N=4)$ for the listed genotypes. SOD knockdown as well as VAP overexpression appears to increase cellular ROS levels. Statistical tests are described in Suppl. table 2.

E: Model depicting the effect of overexpression of wildtype and mutant VAP on ROS. 
bioRxiv preprint doi: https://doi.org/10.1101/368100; this version posted July 13, 2018. The copyright holder for this preprint (which was not certified by peer review) is the author/funder. All rights reserved. No reuse allowed without permission.
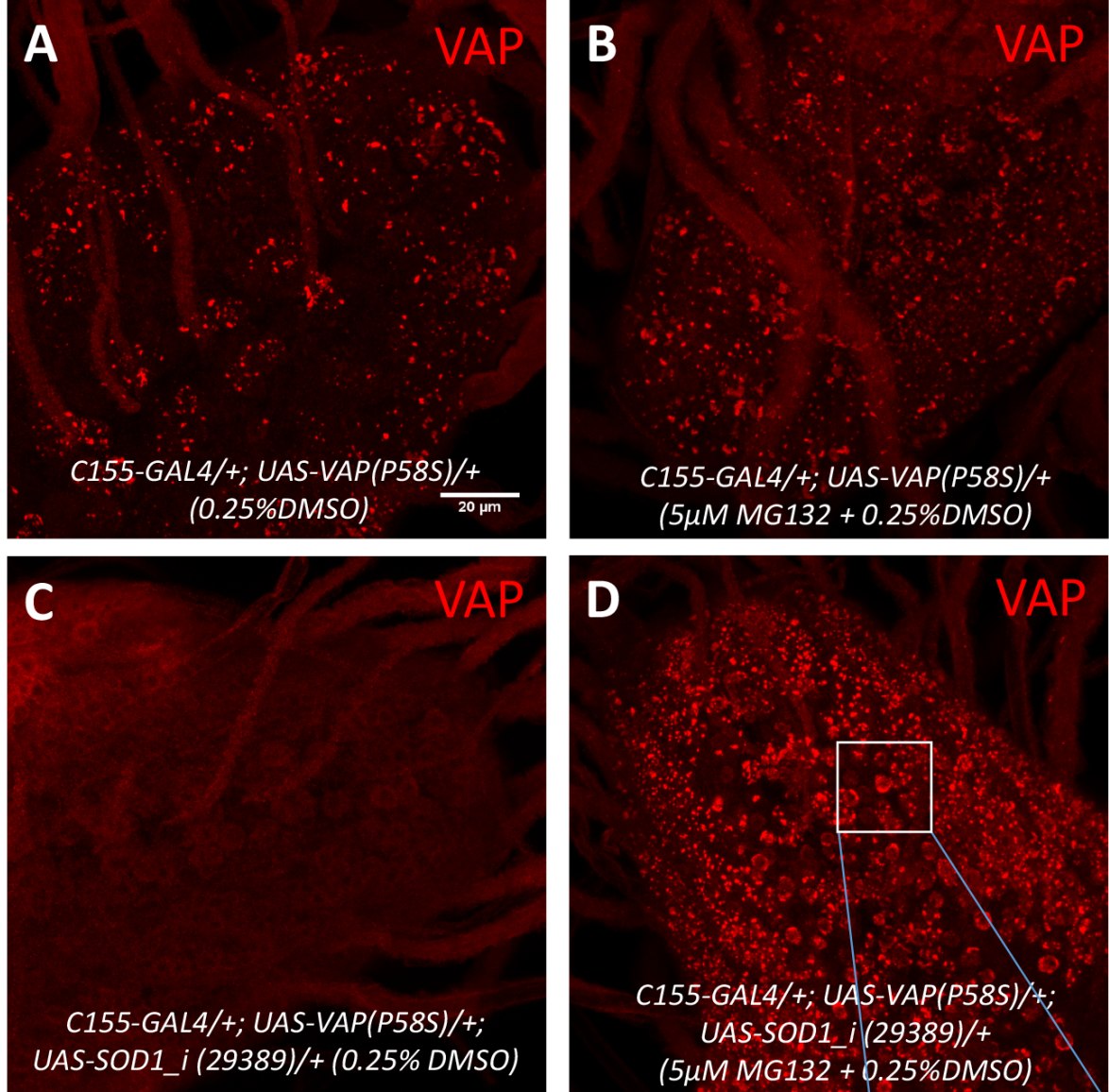

\section{E}
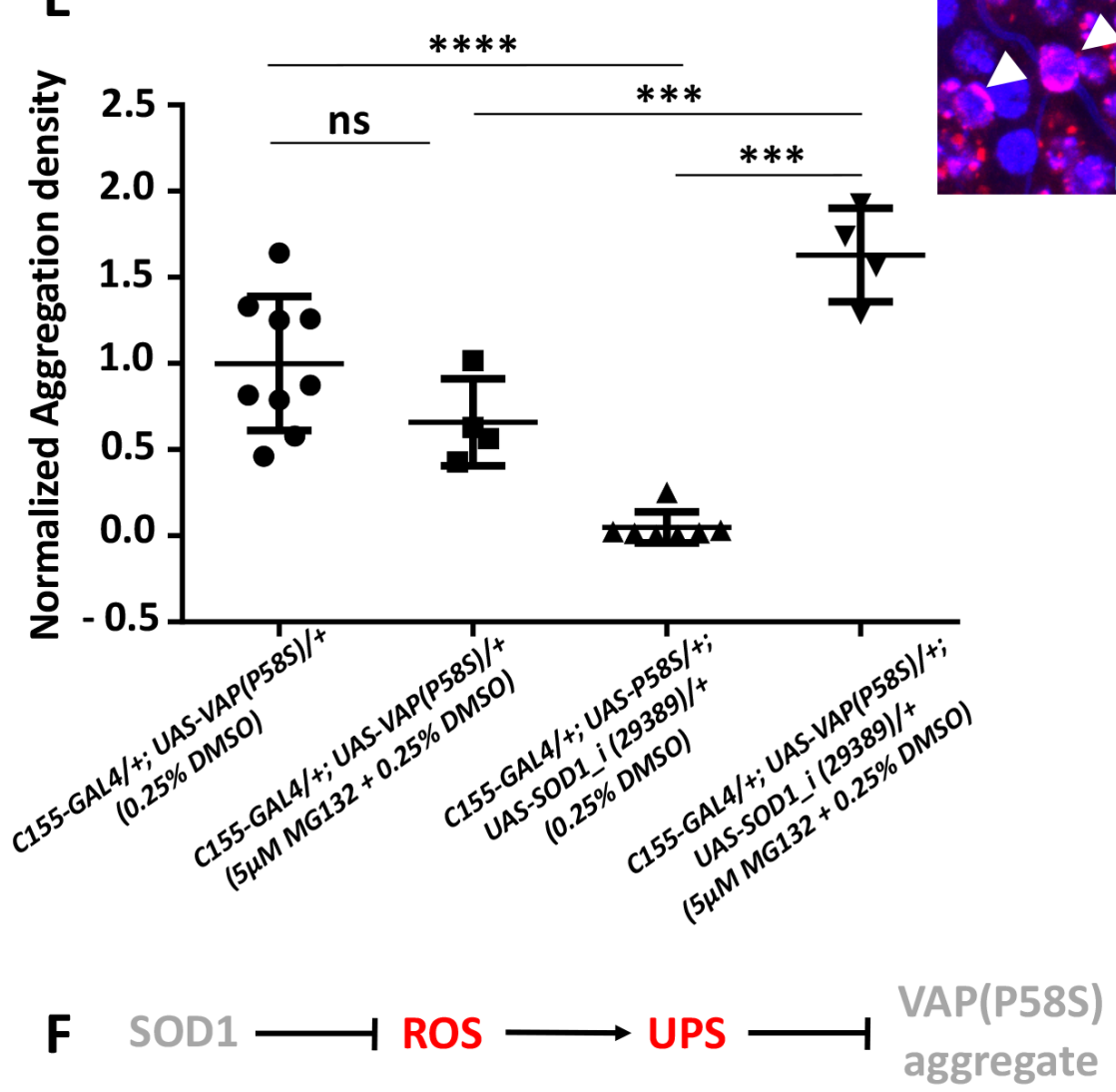

Figure 5: ROS activates proteasomal machinery:

A,B: MG132 feeding of C155GAL4/+; UAS-VAP(P58S)/+, to inhibit proteasomal machinery, does not accumulate aggregation.

C,D,D': MG132 feeding of C155-GAL4/+; UAS$\operatorname{VAP}(P 58 S) /+;$ UAS-SOD1_i (29389)/ $t$, accumulate aggregation. The aggregates, in presence of ROS and MG132, seem to be smaller, scattered and localized around the nuclear membrane (arrowheads) as depicted in inset ( $\left.D^{\prime}\right)$.

E: Plot showing significant decrease in aggregation density in the ventral nerve cord in C155-GAL4/+; UAS-VAP(P58S); UAS-SOD1_i(29389)/+ as compared to C155-GAL4/+; UAS-VAP(P58S)/+ control. This decrease is rescued by feeding $5 \mu \mathrm{M}$ MG132 and is significantly higher than the C155-GAL4/+; UAS-VAP(P58S)/+ control, both unfed and fed with MG132. All images were taken at the same magnification. ANOVA ( $P$ value: $* * * *<0.0001)$ Fisher's LSD multiple comparison test (P-values, $* * *<0.001$, $* * * *<0.0001$ )

F: Model depicting the role of SOD1-regulated ROS in activating proteasomal degradation of VAP(P58S) protein/aggregates. 
bioRxiv preprint doi: https://doi.org/10.1101/368100; this version posted July 13, 2018. The copyright holder for this preprint (which was not certified by peer review) is the author/funder. All rights reserved. No reuse allowed without permission.
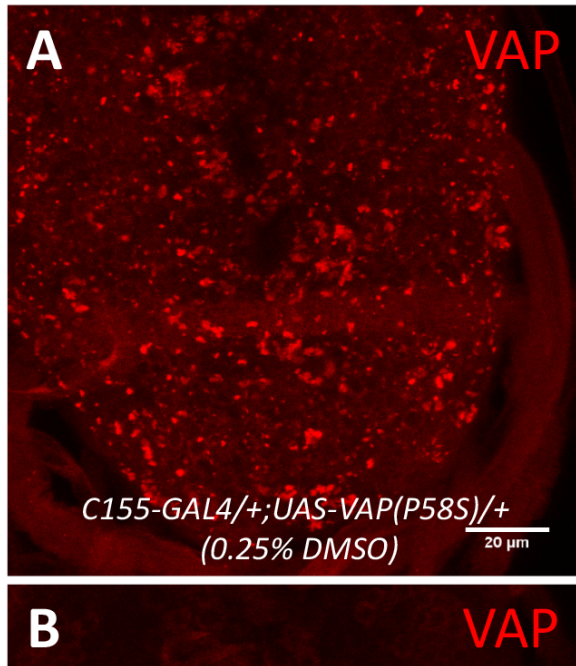

C155-GAL4/+;UAS-VAP(P58S)/+

(200nM Rapamycin +0.25\% DMSO)
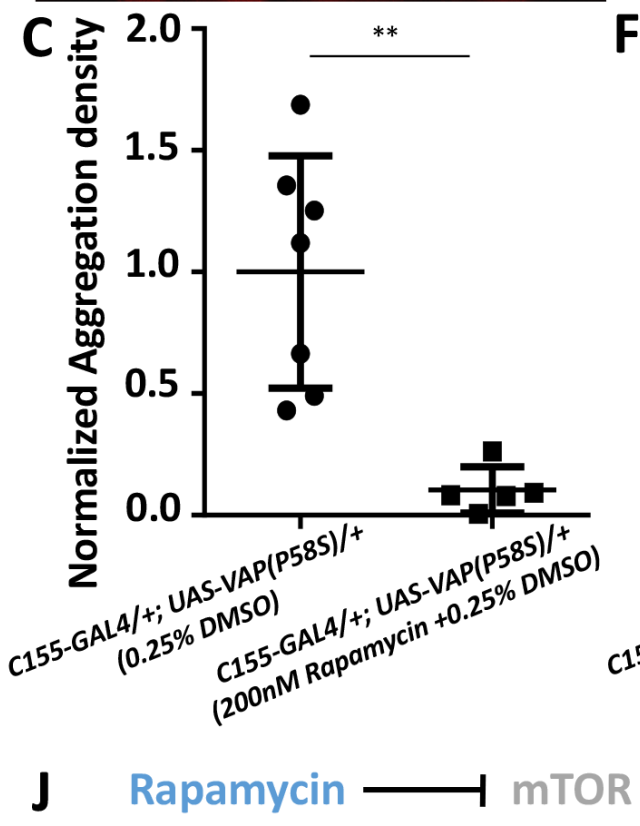
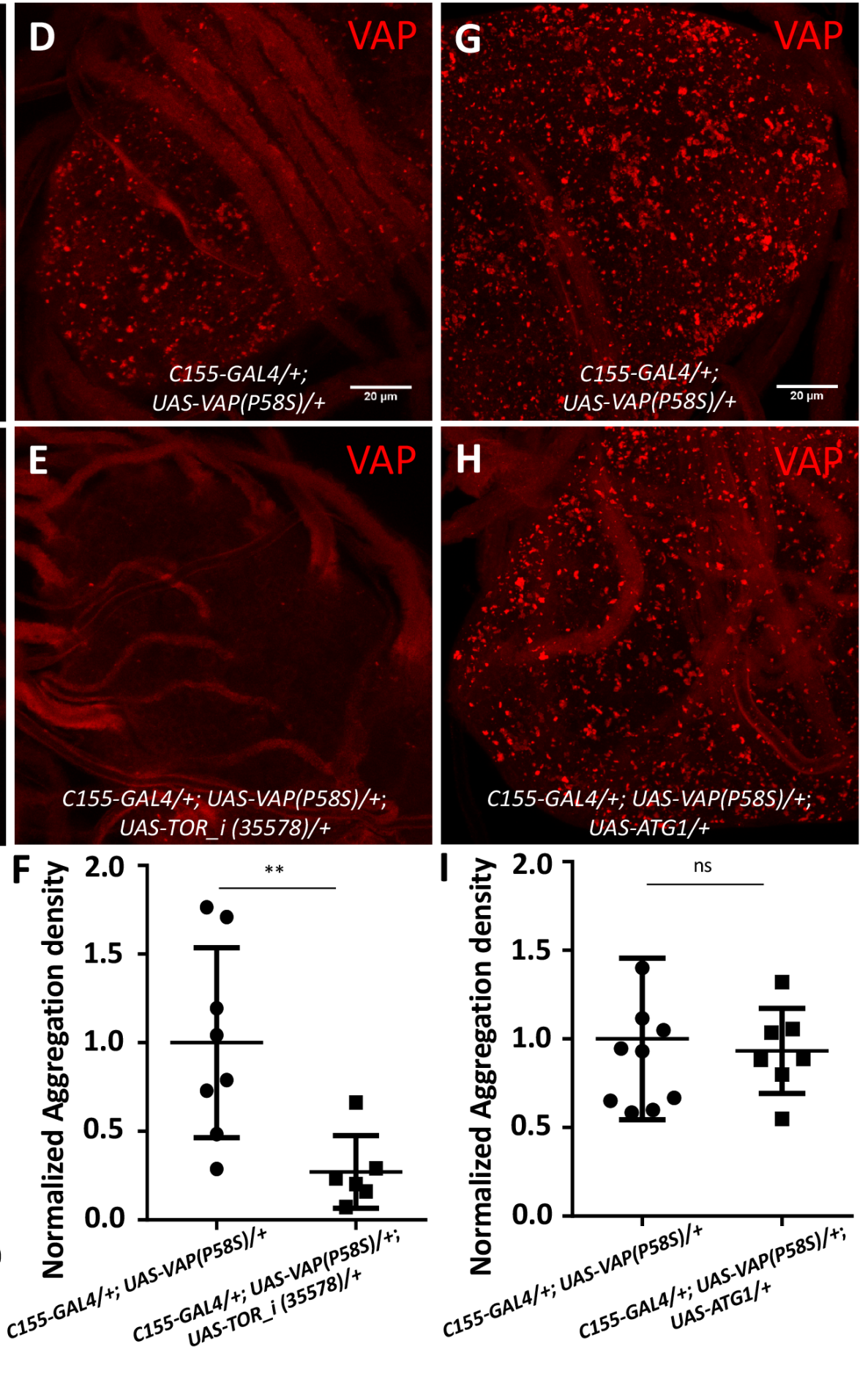

\section{Autophagy $\longrightarrow \sim \begin{aligned} & \mathrm{VAP}(\mathrm{P} 58 \mathrm{~S}) \\ & \text { aggregate }\end{aligned}$}

Figure 6: mTOR inhibition reduces VAP(P58S) aggregation independent of autophagy.

A-C: Rapamycin feeding decreases aggregation density in the ventral nerve cord of third instar larval brains in C155-GAL4/+; UAS-VAP(P58S)/+ flies.

D-F: Neuronal TOR knockdown decreases aggregation density in the ventral nerve cord. The '_ $i$ ' appended to the gene name indicates an RNAi line.

G-I: Neuronal overexpression of Atg1 did not affect the aggregation density in the ventral nerve cord. All images were taken at the same magnification. Students's t-test ( $P$-value, $* *<0.01$ ) J: Model depicting mTOR-regulated clearance of aggregation, independent of autophagy. 
A

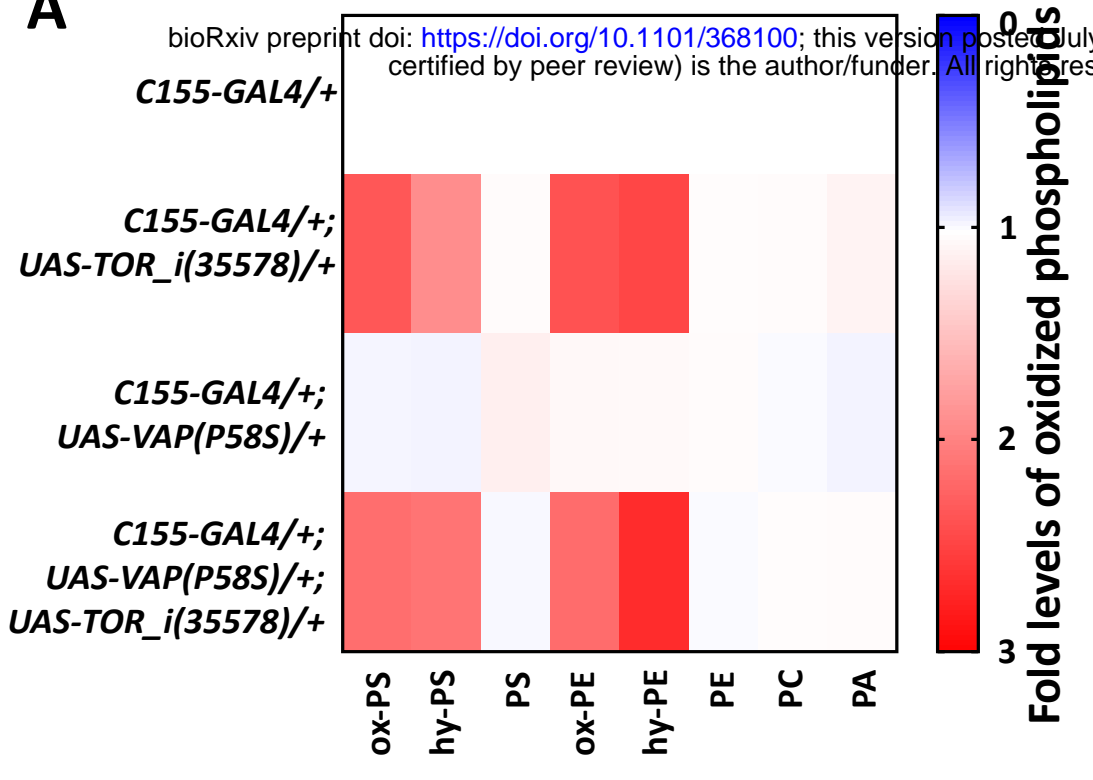

B 13,2018 The copyright holder for this preprint (which was not
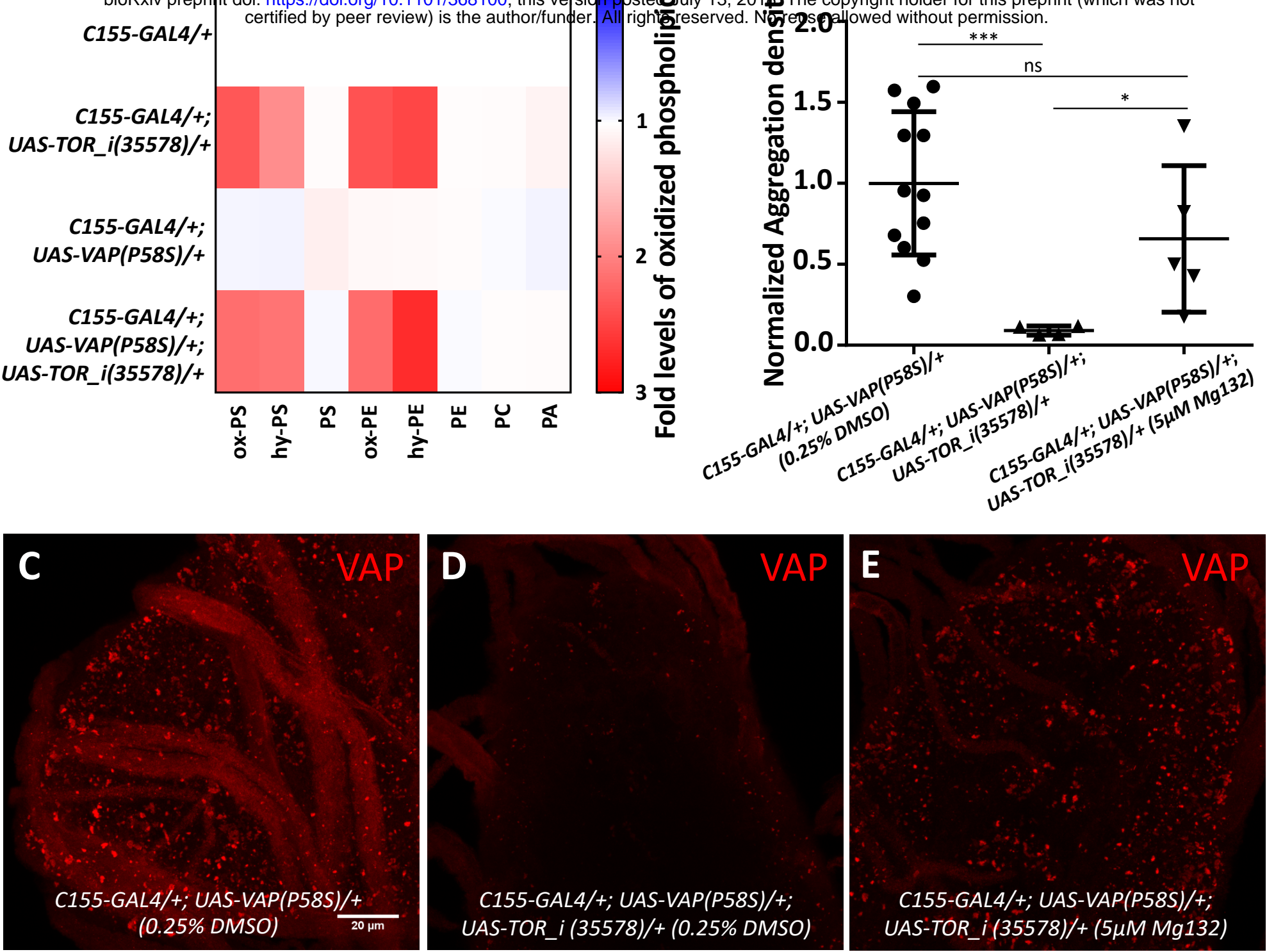

$\mathbf{F}$

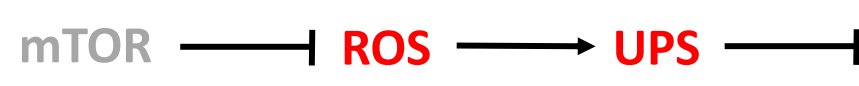

VAP(P58S) aggregate

Figure 7: mTOR inhibition increases ROS leading to proteasomal degradation of VAP(P58S) protein/aggregates:

A: Heat map depicting change in levels of oxidized phospholipids with TOR knockdown normalized to $C 155-G A L 4 /+$, quantified using MS in response to ROS generated in third instar larval brains $(\mathrm{N}=3 / 4)$ for the listed genotypes. Statistical tests are described in supplementary table 2

B: Plot showing significant decrease in aggregation density in the ventral nerve cord in C155GAL4/+; UAS-VAP(P58S); UAS-TOR_i (35578)/+ as compared to C155-GAL4/+; UAS-VAP(P58S)/+ control. This decrease is partially rescued by feeding $5 \mu \mathrm{M} \mathrm{MG132}$. ANOVA (P-value: **, 0.0042) Fisher's LSD multiple comparison test (P-values, $*<0.05, * * *<0.001$ )

C,D,E: Representative images of third instar larval brains showing the partial recovery of aggregates upon $5 \mu \mathrm{M}$ MG132 feeding in C155-GAL4/+; UAS-VAP(P58S)/+; UAS-TOR_i (35578)/+ larvae. All images were taken at the same magnification.

F: Model depicting the role of mTOR-regulated ROS in activating proteasomal degradation of VAP(P58S) protein/aggregates. 


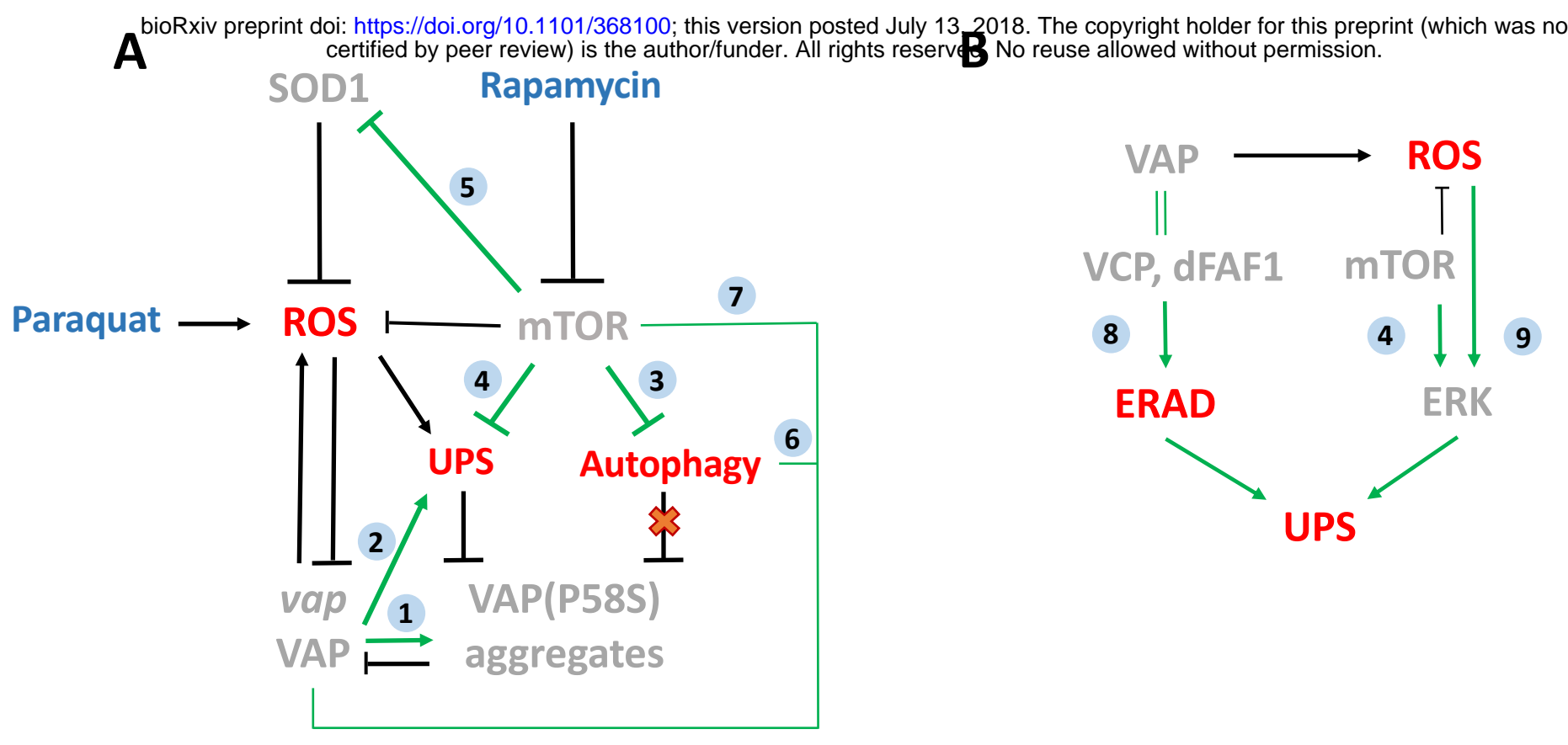

Figure 8: An integrated model for ROS mediated clearance of VAP(P58S) aggregates via UPS.

A: Model depicting novel relationships of SOD1(ALS1) and mTOR- induced ROS with vap and VAP(P58S) aggregates. Clearance of VAP(P58S) protein/aggregates appears to be primarily via the Ubiquitin-Proteosomal system (UPS), triggered by cellular pathways such as mTOR pathway, SOD1 and VAP activity, which in turn regulate ROS. Autophagy does not appear to be a major contributor for aggregate clearance, under the conditions of our experiment.

B: A hypothetical model proposing the possible link between VAP, ROS and UPS. VAP could regulate the UPS via the ERAD pathway due to its interaction with VCP via dFAF1/Caspar. ROS could be the connecting link between mTOR pathway and ERK pathway that together regulate the components of the proteasomal machinery. The link between VAP and ROS that we have demonstrated could modulate proteasomal activity in the cell.

Gray text indicates Genes (italics) and proteins (Capitals); Red text indicates cellular mechanisms; Blue text indicates drugs; Arrows: Black: Experimental evidence, this study; Green: Relationship described in literature; Numbers inside blue circles indicate research papers: 1. Ratnaparkhi et al., 2008; 2. Kanekura et al., 2005; Kuijpers et al., 2013; 3. Noda and Ohsumi, 1998; Perluigi et al., 2015; 4. Zhao et al., 2015; Rousseau et al., 2016; 5. Sun et al., 2012; Tsang et al., 2018; 6. Gomez-Suaga et al., 2017; Zhao et al., 2018; 7. Deivaisigamini et al., 2014; 8. Baron et al., 2014; Papiani et al., 2012; 9. Cavanaugh et al., 2006; Su et al., 2014. 


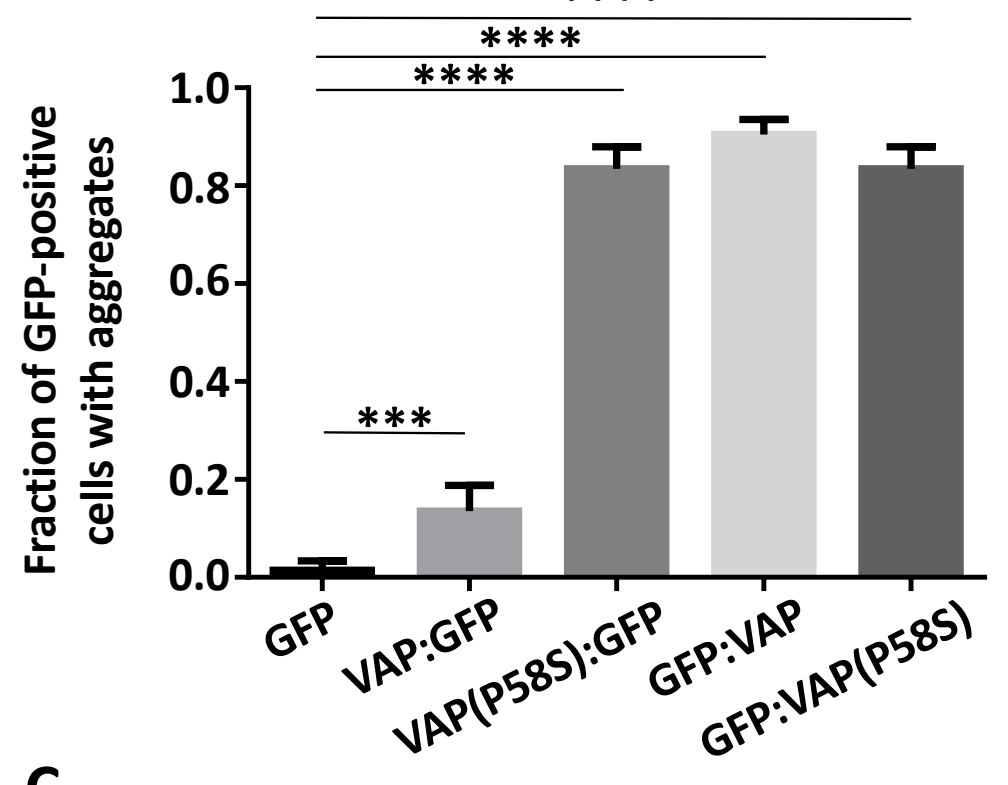

\section{$10 \mu \mathrm{m}$}

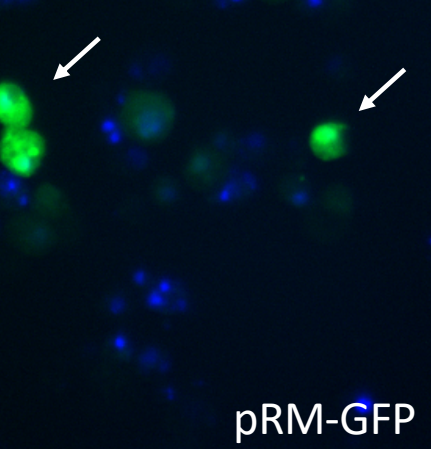

\section{SUPPLEMENTARY FIGURE 1:}

A: Fraction of GFP-positive cells showing aggregates plotted for transiently transfected with $\mathrm{C}$-terminally or $\mathrm{N}$-terminally tagged GFP constructs of VAP or VAP(P58S) and only CFP construct at 24 hours post $500 \mu \mathrm{M} \mathrm{CuSO}_{4}$ induction. Unlike C-terminally tagged VAP, Nterminally tagged VAP forms aggregates as compared to GFP alone. Both $\mathrm{C}$ and $\mathrm{N}$-terminally tagged VAP(P58S) proteins form aggregates. ANOVA (P-value: $* * * *<0.0001)$ Fisher's LSD multiple comparison test (P-values, $* * *<0.001$, $* * * *<0.0001)$.

B: Homogenous cytoplasmic expression of GFP in $\mathrm{S} 2 \mathrm{R}+$ cells.

C: The end result of the screen: a list of 85 genes identified based on total cell intensity as a parameter; these genes are predicted to modify aggregation of VAP(P58S):GFP. Graph displays the percent fold enrichment of targets within each gene category. Genes are listed in Suppl. Table 1D. 


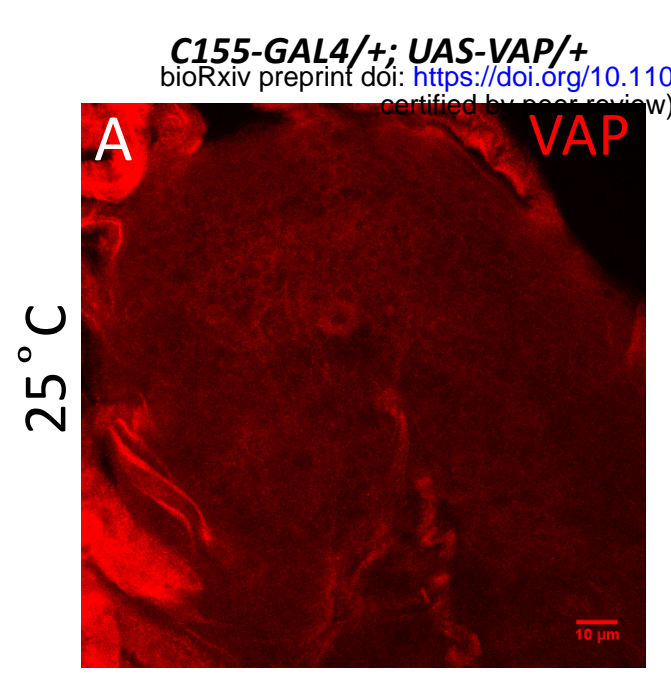

\section{SUPPLEMENTARY FIGURE 2: A} system for measuring VAP(P58S) aggregation in the larval brain. A: Overexpression of VAP in the ventral nerve cord of the third instar larval brain, driven by pan-neuronal C155-GAL4, immunostained with rabbit anti-CCD (VAP) antibody, shows membrane localization. B-D: Overexpression of VAP(P58S) is visualized as inclusions in the third instar larval brains. Temperature dependent increase in aggregation density is seen in the ventral nerve cord in C155-GAL4/+; UAS$\operatorname{VAP}(P 585) /+$ larvae.

E-G: Knockdown of VAP in C155GAL4/+; UAS-VAP(P58S)/+ larvae leads to a corresponding decrease in aggregation density at each temperature.

H: Plot showing significant increase in VAP(P58S) aggregation density with increase in temperature, and a significant decrease in aggregation density in the ventral nerve cord in C155-GAL4/+; UAS-VAP(P58S); UAS$V A P_{-} i(27312) /+$ as compared to C155-GAL4/+; UAS-VAP(P58S)/+ control in a temperature dependent manner.

All images were taken at the same magnification. ANOVA (P-value:

$* * * *<0.0001)$ Fisher's LSD multiple comparison test (P values, ${ }^{*}<0.05$, $* *<0.01, * * *<0.001)$.
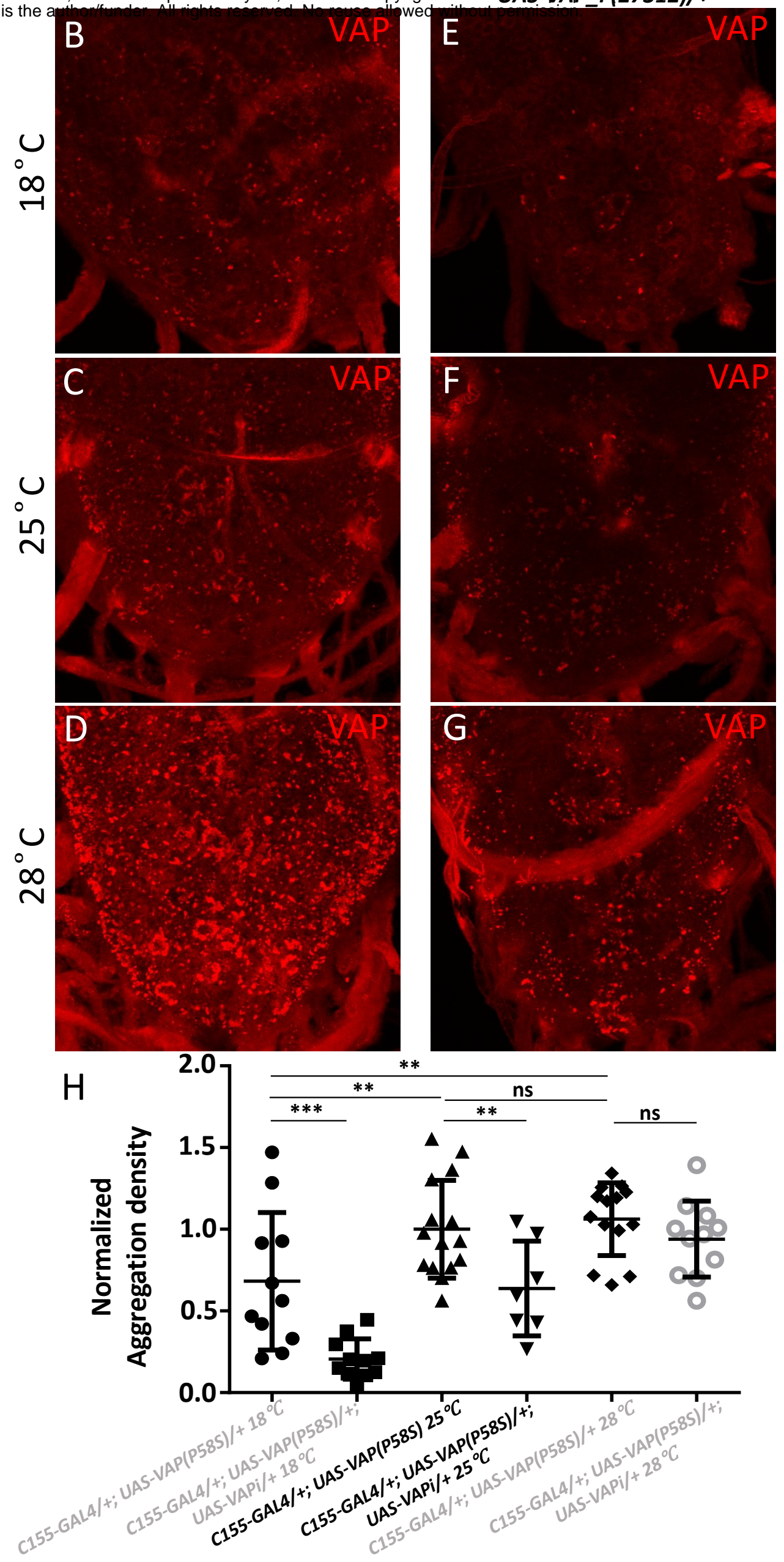


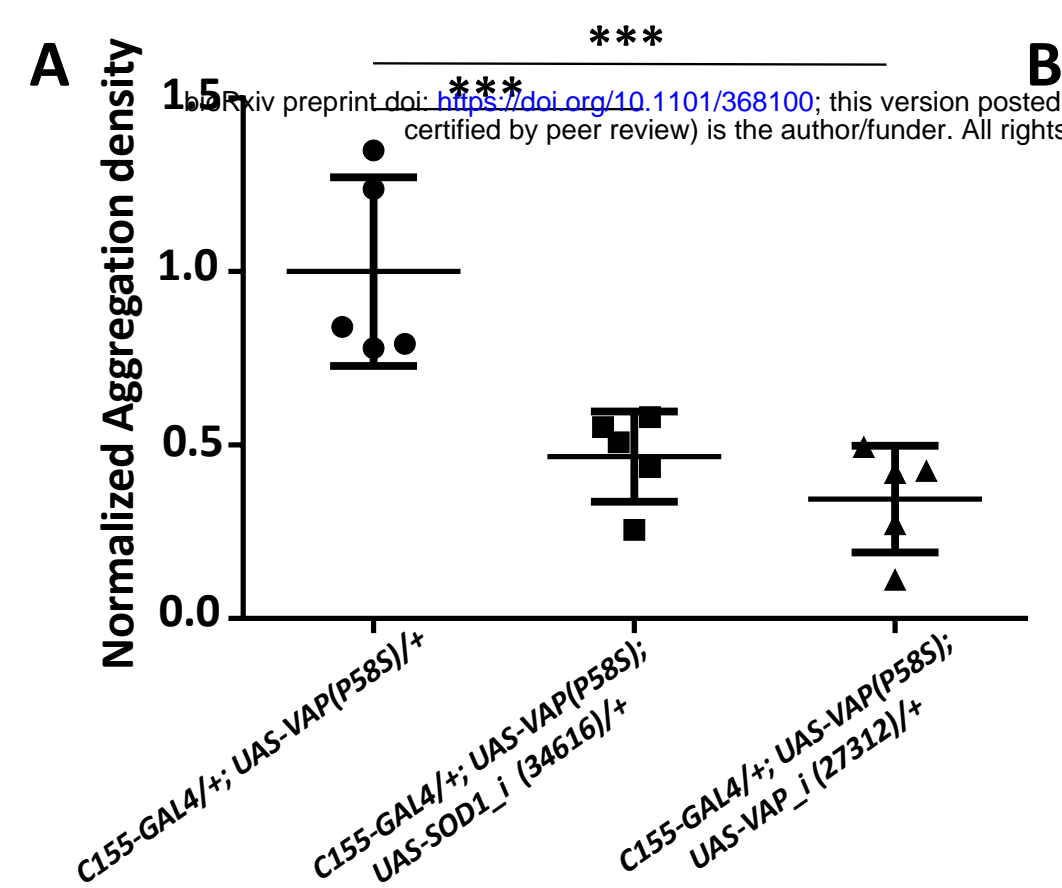

B $\sum_{ \pm=1}$

ns

3, 20 3 . The copyright holder for this preprint (which was not
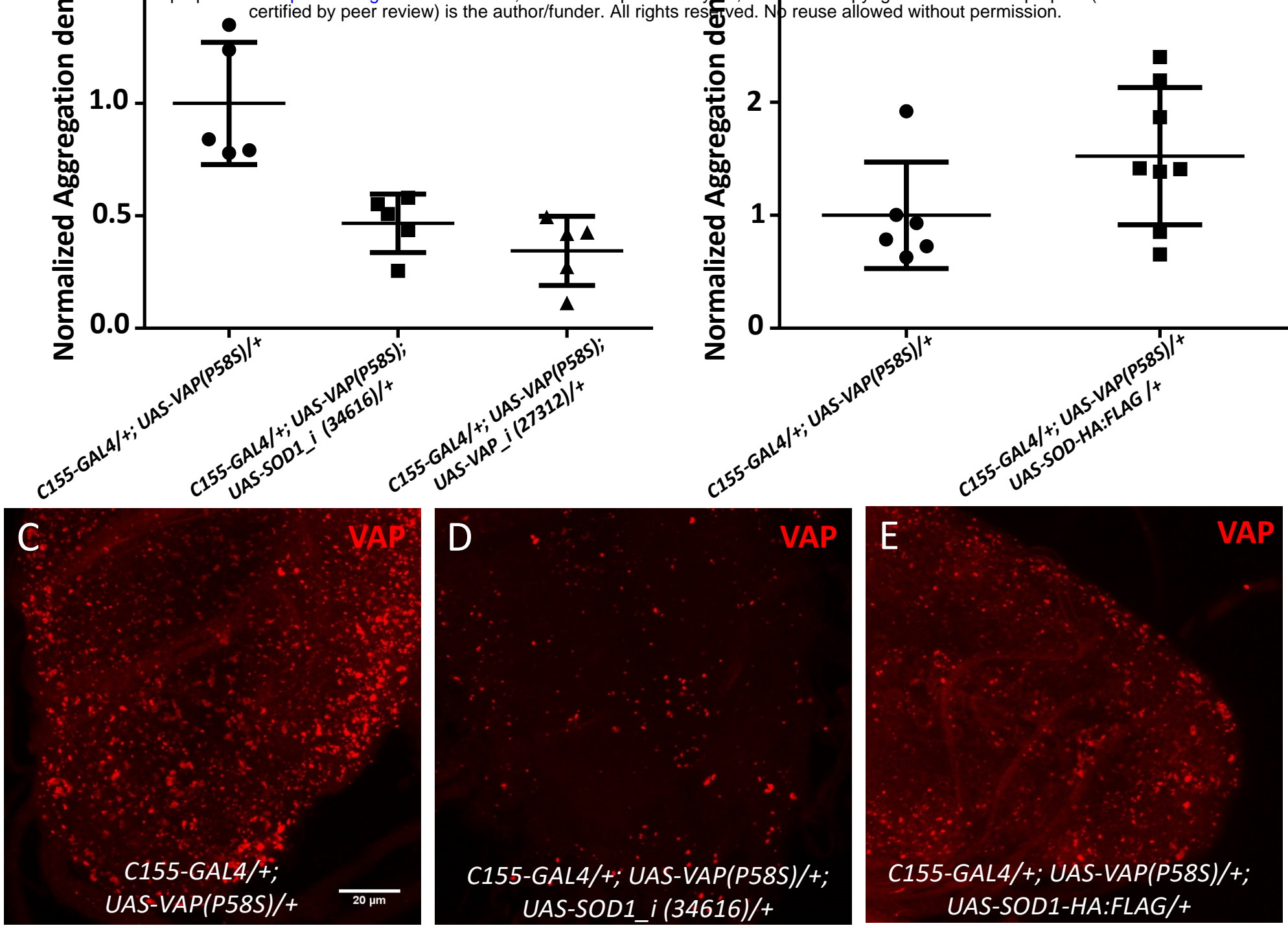

SUPPLEMENTARY FIGURE 3: ROS scavenging genes modulate VAP(P58S) aggregation density in the third instar larval brain

A: SOD1 knockdown decreases aggregation density. ANOVA (P-value ***, 0.0004) Fisher's LSD multiple comparison test ( $\mathrm{P}$-value, $* * *<0.001$ )

B: SOD1:HA:Flag overexpression does not affect aggregation density. Student's t test (P-value: 0.1066) C, D, E: Representative images of the ventral nerve cord showing aggregation of VAP(P58S) (C), with SOD1 knockdown (D), and with SOD1-HA:Flag overexpression (E). All images were taken at the same magnification. F: SOD2 or Catalase knockdown reduces aggregation density. Overexpression of SOD2 does not change aggregation density, however overexpression of Catalase increases aggregation density. The ' $i$ ' appended to the gene name indicates an RNAi line. ANOVA (P-value: $* * * *<0.0001)$ Fisher's LSD multiple comparison test ( $\mathrm{P}$ value, $* *<0.01, * * *<0.001)$.

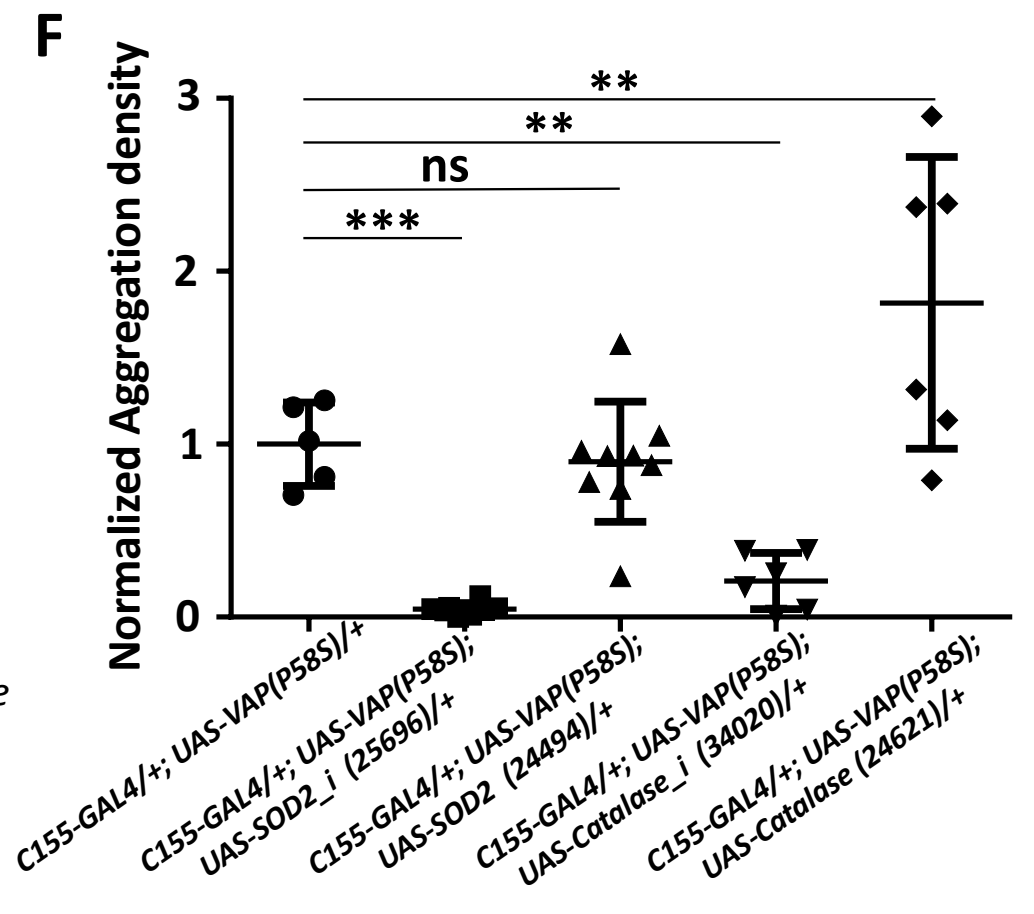


bioRxiv preprint doi: https://doi.org/10.1101/368100; this version posted July 13, 2018. The copyright holder for this preprint (which was not certified by peer review) is the author/funder. All rights reserved. No reuse allowed without permission.

A

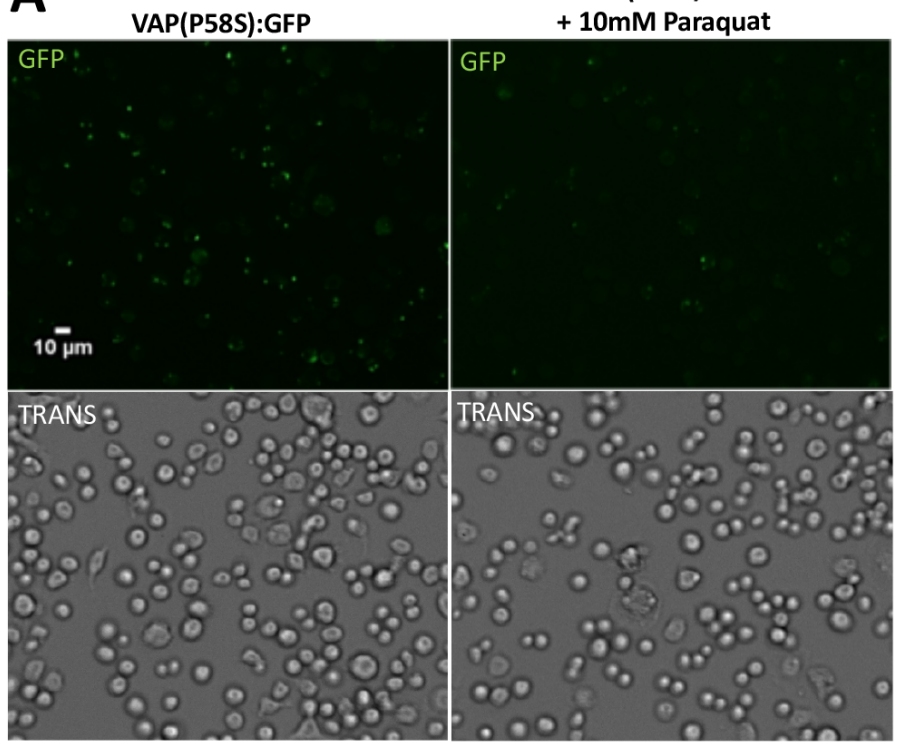

B
C

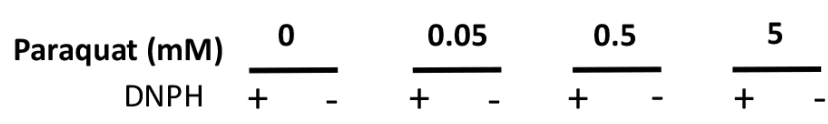

Anti-DNP

D

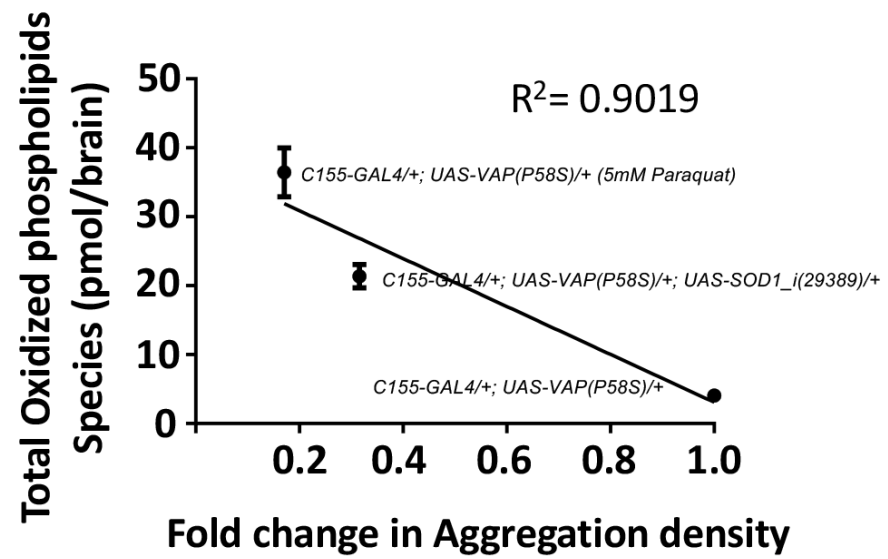

E

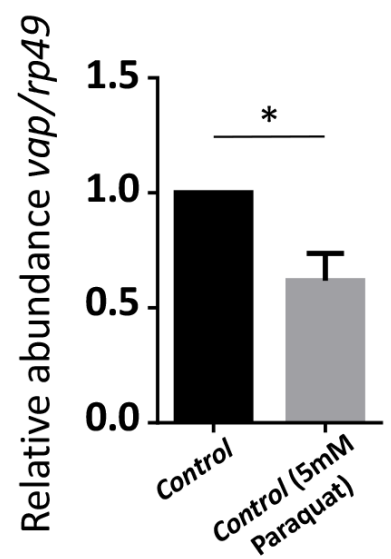

$\mathbf{F}$

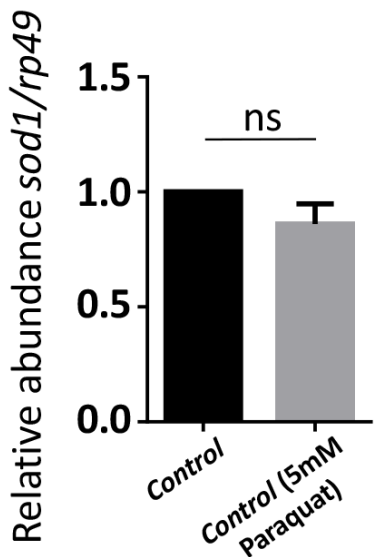

\section{SUPPLEMENTARY FIGURE 4: ROS levels are modulated by SOD1 and VAP and vice-versa.}

A: $10 \mathrm{mM}$ Paraquat treatment for 4 hour, prior to inducing VAP(P58S):GFP in stable S2R+ cell line, reduces the fraction of cells showing aggregation observed 24 hours post-induction.

B: Feeding $5 \mathrm{mM}$ paraquat decreases aggregation density in the ventral nerve cord of third instar larval brains in C155-GAL4/+; UAS-VAP(P58S)/+ flies. All images are taken at the same magnification.

C: Higher levels of protein oxidation in larval brains $(\mathrm{N}=10)$ is seen using Oxyblot, in response to Paraquat feeding. This experiment serves as a calibration/standard for Fig. 4D. Values below the gel indicate fold intensity of the strongest band, when compared to the control ( $0 \mathrm{mM}$ papraquat).

D: Inverse correlation between total oxidized phospholipids and fold change in aggregation density.

E: Relative mRNA levels $V A P$, in the larval brain are lowered on treatment with $5 \mathrm{mM}$ paraquat suggesting that high levels of ROS may negatively regulate VAP transcripts. Student's t test was performed. P-value $* 0.05$

F: Relative mRNA levels of sod1, in the larval brain, do not change in larvae fed with $5 \mathrm{mM}$ paraquat. 